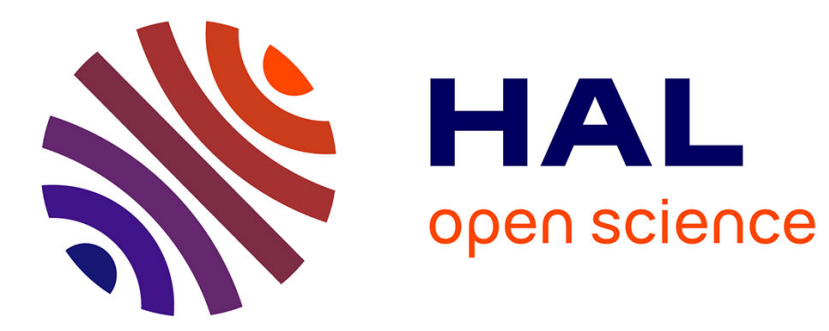

\title{
New framework for parallel interconnection of buck converters: Application to optimal current-sharing with constraints and unknown load
}

Jean-François Trégouët, Romain Delpoux

\section{- To cite this version:}

Jean-François Trégouët, Romain Delpoux. New framework for parallel interconnection of buck converters: Application to optimal current-sharing with constraints and unknown load. Control Engineering Practice, 2019, 87, pp.59-75. 10.1016/j.conengprac.2019.03.003 . hal-02127803

\section{HAL Id: hal-02127803 \\ https://hal.science/hal-02127803}

Submitted on 22 Oct 2021

HAL is a multi-disciplinary open access archive for the deposit and dissemination of scientific research documents, whether they are published or not. The documents may come from teaching and research institutions in France or abroad, or from public or private research centers.
L'archive ouverte pluridisciplinaire HAL, est destinée au dépôt et à la diffusion de documents scientifiques de niveau recherche, publiés ou non, émanant des établissements d'enseignement et de recherche français ou étrangers, des laboratoires publics ou privés.

\section{(ㄷ)(1) $\$$}

Distributed under a Creative Commons Attribution - NonCommercial| 4.0 International 


\title{
New Framework for Parallel Interconnection of Buck Converters: Application to Optimal Current-Sharing with Constraints and Unknown Load.
}

\author{
Jean-François Trégouët ${ }^{\mathrm{a}}$, Romain Delpoux ${ }^{\mathrm{a}}$ \\ ${ }^{a}$ Laboratoire Ampère CNRS UMR 5005, Université de Lyon, INSA - Lyon, 25 avenue Jean Capelle, 69621 Villeurbanne, \\ France (e-mail: jean-francois.tregouet@insa-lyon.fr).
}

\begin{abstract}
In this paper, heterogeneous set of parallel buck converters, feeding an unknown resistive load via a common DC bus, is considered. When addressing control design problem of this interconnected power converter, one has to accommodate competing objectives of (i) voltage regulation and (ii) power distribution among branches, also called current-sharing in this context. Related dynamics being coupled, classical solution resorts to frequency separation to prevent undesirable interaction between them. In stark contrast with this approach, this paper proposes a novel framework which completely separate voltage regulation from current distribution dynamics without frequency separation, hence offering tractability without sacrificing performance. Such a reformulation is performed on the open-loop model so that control law candidates are not confined in some particular class. Arbitrary large set of heterogeneous converters can be handled. Remarkably, voltage regulation boils down to the control of a single virtual buck converter. Controller design example exploiting the new structure is provided. In the context of unknown resistive load, this controller achieves voltage regulation and minimizes overall power losses at the steady-state while taking current limits of each branch into account. Those results are supported by formal statements and proofs, and assessed through experimental results.
\end{abstract}

Keywords: Power-system control, Parallel power converters, DC-DC converters, Current-sharing, Multi-port converters.

\section{Introduction}

\subsection{About parallel interconnection of converters}

Substituting a single high-capacity centralized electrical power converter by multiple distributed converters connected in parallel is a strategy that becomes more and more popular. Indeed, paralleling converters offers several advantages such as increased reliability due to redundancy and distribution of stresses of components, ease of maintenance and repair, improved thermal management and reduced output ripple by interleaving phase of Pulse Width Modulation (PWM) $[1,2,3]$.

An essential feature offered by parallel interconnection of converters is the possibility to distribute load current. Indeed, if regulation of output voltage imposes overall current, distribution of current among 

sharing which uniformly distributes currents among converters, see e.g. [1, 2]. If identical converters (same class of converters sharing the same electrical components) are considered, this strategy is fully justified by the fact that it equally distributes stresses among converters.

Vast majority of existing results on this topic have been derived in the context of identical converters. However, several papers demonstrate that making non-identical converters working in concert would improve the unloading transient response and reduce the output voltage overshoot $[4,5,6]$. In the patent [7], asymmetric inductors in multi-phase DC/DC converters are proposed to achieve fast transient response and to optimize efficiency over the load range. Note, however, that classical balanced currentsharing should be reconsidered in this context. Indeed, it has been recently shown that this policy cannot be optimal for all load with respect to overall power losses [8].

On the basis of the previous considerations, this paper aims contributing to the control counterpart of interconnection of nonidentical converters.

\subsection{On the related control problem}

Few papers in the literature consider control of interconnection of non-identical converters and, hence, seeks nonuniform current-sharing at the steady-state. Most of existing solutions achieving such a current distribution rely on "virtual droop resistors" which can be interpreted as a low-frequency negative feedback on current which aims adjusting equilibrium current $[9,10]$. Then a rule of thumb is to fix the droop resistor magnitude for bus voltage to remain into allowed bounds when the converter injects its maximum power [11,9]. As noticed in [10], this strategy maintains constant current ratio between 30 converters. Yet, this prevents current-sharing minimizing power losses to be achievable. Theoretical and experimental proof of this fact has been provided in $[8,12]$, together with optimal control law imposing load dependent current ratio at the steady-state.

Solution proposed in [8] suffers from two limitations, though. First, steady-state current constraints cannot be taken into account. Controller addressing this issue is yet highly desirable since violating those constraints might overheat coils. Second, stability is achievable under some frequency separation argument.

Let us now comment on this second limitation. When dealing with parallel converters, both voltage and current distribution have to be regulated. It is well known that control of both related dynamics may badly interact and, in turn, induce closed-loop instabilities if not properly treated [1, 2]. This phenomena

40 can be experienced either when homogeneous or heterogeneous set of converters is considered. Controlling parallel converters, and therefore dealing with the above (possibly interacting) dynamics, has been a topic of extensive research effort well surveyed in $[2,13]$ for the homogeneous case. Remarkably, almost all existing solutions make use of a two nested loops scheme and resort to some frequency separation argument. Bandwidth of voltage regulation (the outer loop) is typically narrower than that of current distribution control (the inner loop) [2]. By contrast, outer loop proposed in [8] performs fine voltage and current distribution regulation with comparable transient duration. However, it relies on low frequency 
model of some closed-inner-loop which prestabilized the system, so that frequency separation remains the key to achieve stability.

If this strategy offers tractability, it also unavoidably lowers performance: Transient of the outer loop has to be sufficiently slow not to destabilize the inner loop. Relying on models with complex impedances, interesting research directions have been opened to go beyond this frequency separation: In [1] arbitrary number of identical converters are considered whereas the case of two different converters is treated in [14]. Theoretical stability certificates of the overall system are not formally provided, though. Furthermore, control schemes (the so-called "master/slave" and "democratic") are a priori imposed even if discussion about their intrinsic conservatisms seems hard to handle. In addition to that, generalization to arbitrary number of non identical converters along those lines leads to calculus burden.

We believe that limitations of most of those existing results are inherent to the retained methodology which heavily relies on interconnection of single input single output (SISO) transfer functions. In this paper, we seek a different strategy which rather adopts multi-variable point of view, allowing for geometric decomposition of both state and input spaces.

\subsection{Scope and contributions of the paper}

Parallel interconnection of arbitrary number of buck (step-down) DC/DC converters having distinct characteristics is considered in this paper. Resulting electrical circuit is depicted by Fig. 1 considering $m$ branches.

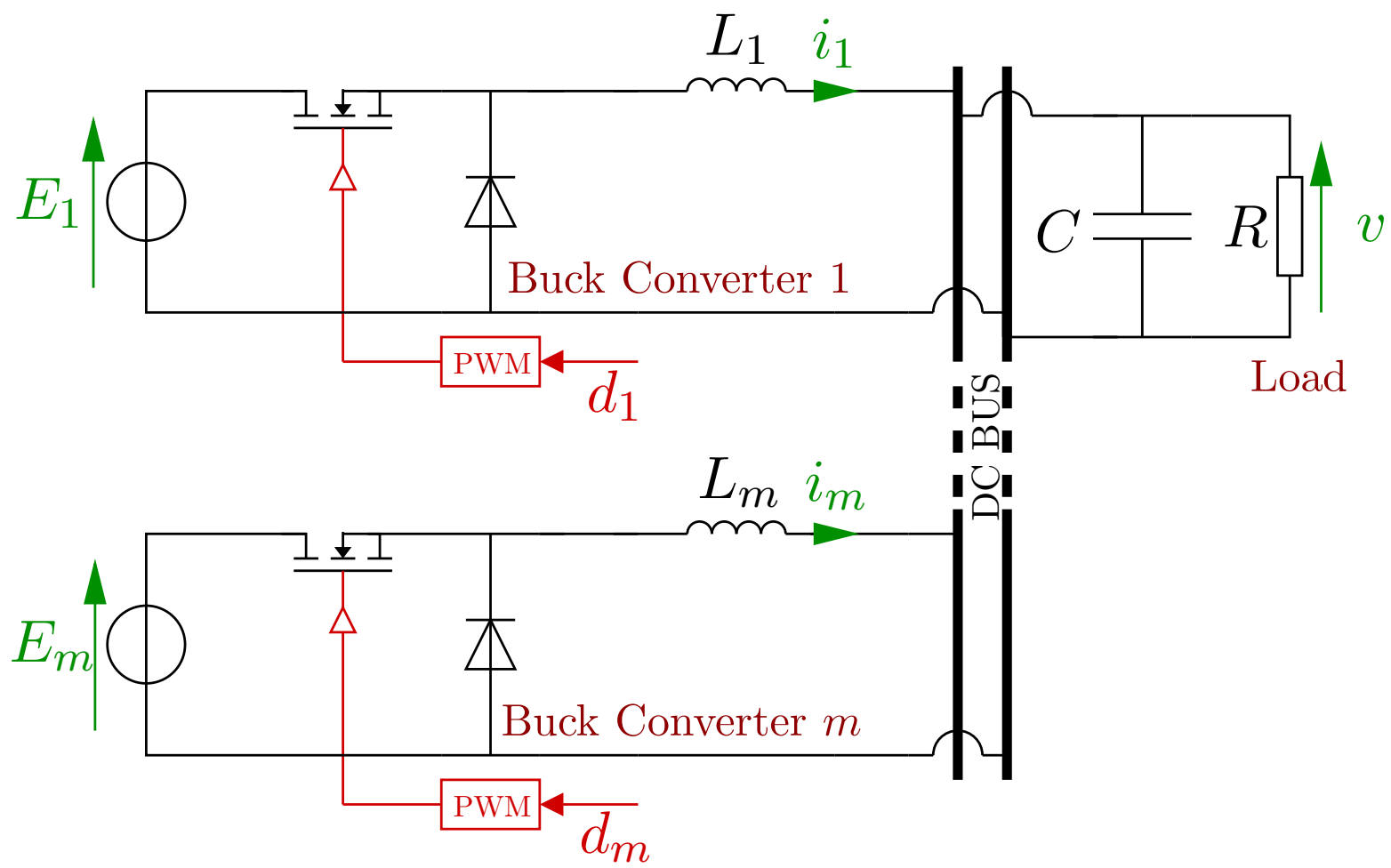

Figure 1: Electrical schematic. 

$m$ inputs (duty cycles) to $m+1$ outputs (currents and output voltage) model, a batch of disconnected SISO subsystems is constructed. The two formulation being equivalent, it is shown that the original control problem reduces to independent feedback subdesigns related to mere SISO models. Let us already expose benefits of the proposed framework, as compared with classical existing solution.

No frequency separation assumption. Perhaps, the most remarkable feature of the novel framework is to completely separate voltage regulation from current distribution dynamics, hence preventing undesirable interaction between them either during transient or at steady-state. No frequency separation assumption being required, this new scheme offers tractability without sacrificing performance. This suggests that the two related control objectives are actually not competing if properly treated.

Broad domain of applicability. This new framework possesses remarkable universality properties. (i) Arbitrary large set of heterogeneous converters can be handled, i.e. electrical components of each branches can be freely selected. This is in contrast with most of existing control solution which relying on the assumption of strictly identical branches. (ii) Whereas most of existing control solution are concerned with balanced current-sharing, arbitrary and load-dependent current-distribution can be easily considered as control objective. This makes the new scheme suitable for heterogeneous set of converters for which balanced current-sharing is not optimal with respect to power losses [12]. (iii) Dynamical model associated with this new framework are constructed by manipulated open-loop equations, instead of some precompensated model. As a result, control law candidates are not restricted to the ones compatible with preselected inner controller. To put it another way, every control scheme solving the control problem

85 can be reformulated into the proposed framework.

Suitable structure for control design. Formulation of the new framework allows simple controller derivation. (i) Adopting the form of a cascaded scheme, it allows for standard tools related to this dynamical structure to be used and, in turn, formal stability proofs to be more easily derived. (ii) Circuit theory interpretation shows that voltage regulation boils down to classical output voltage control of a single virtual buck converter whereas current distribution assignment is nothing but a set of independent regulation problem of current flowing through a single virtual coil connected to a controlled voltage source. This interpretation allows designers to make use of physical intuition throughout design procedure.

\subsection{Outline and notations}

The paper is structured as follows. In Section 2, we formalize our problem statement and introduce a suitable preliminary assumption for the problem to be well-posed. Model description is provided and a running example of three bucks serving didactic purpose throughout this paper is proposed. In Section 3, the classical solution of nested loops is reviewed. Its underlying assumption about frequency separation between the loops is discussed and highlights via simulations. In Section 4, open-loop equations are manipulated to arrive at our main contribution, which significance is discussed. In Section 5, an example 
of control design, whose structure is naturally suggested by the new framework, is provided. In Section 6 , performance of this controller is assessed via the physically relevant scenario where closed-loop system has to asymptotically converges to the set-point regulating output voltage as well as minimizing overall power losses while sticking into admissible power limits associated with each branch. Finally, in Section 7 we discuss relevance of the new framework to cop with untreated difficulties like constant-power load, measurement failure and input saturation.

A preliminary version of this paper was presented in [15]. Here, as compared to [15], we propose an extensive discussion on the pervasive two-loops scheme considered in the literature (Section 3). We also include statements and proofs of the stability property of the proposed controller (Section 5.2). In addition to that, proposed design strategy is illustrated throughout the paper by means of a running example (see Subsection 2.5 and "Example" environments), and assessed via experiments tackling a scenario which is meaningful from power electronics point of view (Section 6).

Notation: The symbol $\mathbf{I}_{m}$ stands for the identity matrix of dimensions $m \times m$. The null matrix of size $m \times n$ is denoted by $\mathbf{0}_{m \times n}$. The vector (column matrix) of size $m$ for which every entry is 1 (0) is denoted by $\mathbf{1}_{m}\left(\mathbf{0}_{m}\right)$. The notation $x_{k}$ refers to the $k$-th element of the vector $x$, with 1 being the index of the first element. The operator 'diag' builds diagonal matrix from entries of the input vector argument.

Nomenclature: Main variables introduced in this paper are listed in Table 1.

\begin{tabular}{|c|c|}
\hline$v$ & Voltage bus \\
\hline$i_{k}$ & Current in the $k$-th branch \\
\hline$d_{k}$ & Duty cycle of the $k$-th branch \\
\hline$E_{k}$ & Voltage input of the $k$-th branch \\
\hline$L_{k}$ & Inductance of the $k$-th branch \\
\hline$R \in \mathbb{L}$ & Load magnitude and its domain \\
\hline$C$ & Output capacitor \\
\hline$m$ & Number of branches \\
\hline$i_{r}(R)$ & Load-dependent current reference \\
\hline$v_{r}$ & Voltage reference \\
\hline$\sigma$ & Sum of individual currents, see (2c) \\
\hline$\delta$ & Part of the new state vector, see (5) \\
\hline$T$ & Transformation matrix, see Section 4.1 \\
\hline$\lambda_{k}, \mu$ & Entries of the new input vector, see (11) \\
\hline$L_{\mathrm{eq}}$ & Equivalent inductance, see Section 4.1 \\
\hline$E_{\text {eq }}$ & Equivalent voltage source, see Section 4.1 \\
\hline
\end{tabular}




\section{Problem statement and model description}

This section aims formalizing problem considered in this paper, as well as provides equations governing dynamical model.

\subsection{State-variable and related dynamics}

The electrical circuit represented by Fig. 1 is considered. It corresponds to parallel interconnection of $m$ buck converters sharing a single capacitor and connected to a common resistive load $R$. Magnitude of the load is supposed to be constant and to belong to the compact and connected set

$$
\mathbb{L}:=\left[\begin{array}{ll}
\underline{R} & \bar{R}
\end{array}\right] \subset \mathbb{R}_{>0}
$$

In addition to that, it is emphasized that $R$ is unknown, as in most of practical situations.

Converters are controlled via PWM and $d_{k}$ refers to duty cycle of $k$-th converter where $k$ belongs to the following set

$$
\mathcal{K}:=\{1, \ldots m\}
$$

Voltage of DC bus is denoted by $v$ and current in $k$-th inductor $L_{k}$ is referred to as $i_{k}$. As shown by next section, state vector $x$ of the related model gathers those signals:

$$
\mathbb{R}^{m+1} \ni x:=\left[\begin{array}{ll}
i^{\top} & v
\end{array}\right]^{\top}
$$

Magnitude of voltage sources $E_{k}$ are supposed to be known and constant. Capacitor $C$ is connected in parallel to the load.

Bus voltage regulation to a given value $v_{r} \in \mathbb{R}_{>0}$ represents the foremost control goal. ${ }^{1}$ Nonetheless $v$ only depends on the total current, that is the sum $\sigma$ of each $i_{k}$. Thus, additional degrees of freedom remain in the way $\sigma$ is distributed among converters. For this reason, current distribution control (also called "current-sharing control") is considered as an additional control objective so that power-flow reference through the interconnection is fully defined. Yet, dynamics related to those two objectives are intrinsically coupled, so that particular care should be taken not to jeopardize one of them when addressing the other.

\subsection{General formulation of current-sharing problem}

Typically, one seeks uniform current distribution, also called balanced current-sharing, where steadystate currents of each branch are identical. Since sum of currents must equal $v_{r} / R$ at the steady-state, uniform current distribution requires $i_{k}$ to converge to $v_{r} /(m R)$. As a result, control problem reads as follows: Design a state-feedback control law $(i, v) \mapsto d$ such that, for all load $R \in \mathbb{L}$, voltage $v(t)$ asymptotically tends to $v_{r}$ and $i_{k}(t),(k \in \mathcal{K})$ converges to $v_{r} /(m R)$. Whenever identical converters

\footnotetext{
${ }^{1}$ Note that buck converter is a device that can only reduce input voltage so that each $E_{k}$ must be larger than voltage reference $v_{r}$
} 
are considered, balanced current-sharing strategy is fully justified by the fact that it equally distributes stresses among converters. This policy has to be reconsidered if interconnection of non-identical converters is addressed, though. Indeed, it has been recently shown in [8] not only that balanced current-sharing cannot be optimal for all load with respect to overall power losses but also that optimal current ratio (i.e. proportion of the total current that is to be affected to the each branch) is in general load-dependent. ${ }^{2}$

Motivated by this analysis, this paper aims addressing the general problem where current vector reference $i_{r}$ can arbitrarily depend on $R$. This set-up allows set-point $\left(i_{r}(R), v_{r}\right)$ to be tracked to be selected beforehand via optimization problem of the following kind:

$$
\min _{i} J(i) \text { s.t. } v=v_{r},\left.\frac{d i_{k}}{d t}\right|_{k \in \mathcal{K}}=\frac{d v}{d t}=0 .
$$

Here, cost function $J$ translates current distribution preferences. Section 6 provides a physically meaningful example along this line, by defining $J$ as total power losses and adding inequality constraints to exclude undesirable power flow at the steady-state. Load magnitude $R$ acting as a parameter in this optimization problem, the resulting current reference vector $i_{r}$ minimizing $J(i)$ is indeed a function of $R$. Note that if voltage sources correspond to generator or energy storage devices, then $J$ can also incorporate preferences on power-flow inside the interconnection.

Problem 1. Given a reference profile $x_{r}(\cdot):=\left(i_{r}(\cdot), v_{r}\right): \mathbb{L} \rightarrow \mathbb{R}^{m} \times \mathbb{R}_{>0}$, design (load independent) state-feedback control law $x \mapsto d$ such that, for all constant but unknown $R \in \mathbb{L}$, closed-loop system admits $x_{r}(R)$ as a globally asymptotically stable (GAS) equilibrium.

Remark. From previous discussion, it should be clear that the proposed framework encompasses balanced current-sharing (uniform current distribution) as the particular case for which $x_{r}(\cdot)$ is selected as $\left(v_{r} /(m R), \ldots, v_{r} /(m R), v_{r}\right)=\left(\mathbf{1}_{m} v_{r} /(m R), v_{r}\right) \in \mathbb{R}^{m+1}$.

If letting $i_{r}(R)$ to be load-dependent has clear technological motivation, it is emphasized that complying with this feature makes controller design non trivial as load $R$ is unknown.

\subsection{A necessary condition}

For this problem to admit solutions, it is clear that prescribed $x_{r}(R)$ must be a admissible equilibrium, that is there exists an input vector $d$ that imposes $\dot{x}$ to be equal to zero at $x_{r}(R)$. Remarking that constant voltage equilibrium are such that the sum of currents of each branch equals $v / R$ and that

$$
\sum_{k \in \mathcal{K}} i_{k}=v / R \Leftrightarrow \mathbf{1}_{m}^{\top} i-v / R=0 \Leftrightarrow\left[R \mathbf{1}_{m}^{\top}-1\right] x=0,
$$

it comes out that $x_{r}(R)$ must satisfied the following assumption for the problem to admit solutions.

\footnotetext{
${ }^{2}$ Interested reader can consult [8], where an example of parallel interconnection of two non-identical buck converters is considered. It is proved, both theoretically and experimentally, that for lower output power most of the power should be convey by the second converter, whereas the opposite ordering should to be preferred for higher output power.
} 
Assumption 1. It holds

$$
x_{r}(R)=\left[i_{r}^{\top}(R), v_{r}\right]^{\top} \in \operatorname{Ker}\left\{\left[R \mathbf{1}_{m}^{\top} \quad-1\right]\right\}
$$

for all $R \in \mathbb{L}$.

\subsection{Model description}

Throughout this paper, it is assumed that (i) the Pulse Width Modulation (PWM) switching frequency $f_{s}$ is sufficiently large for the dynamics to be approximated by an average (ripple-free) continuoustime model, (ii) converters remain in continuous conduction mode and (iii) electrical components are ideals, i.e. parasitic resistances can be neglected.

By virtue of Kirchhoff's circuit laws, under previous assumptions, dynamics of the circuit depicted by Fig. 1 is governed by

$$
\begin{aligned}
\forall k \in \mathcal{K}, L_{k} \frac{d i_{k}}{d t} & =-v+E_{k} d_{k}, \\
C \frac{d v}{d t} & =\sigma-v / R,
\end{aligned}
$$

where

$$
\sigma:=\sum_{k \in \mathcal{K}} i_{k}=\mathbf{1}_{m}^{\top} i
$$

refers to the total current. Eq. (2a) describes dynamics of output currents produced by each converter whereas $(2 \mathrm{~b})$ corresponds to output voltage dynamics. The corresponding matrix formulation of previous dynamical equations is as follows:

$$
\left[\begin{array}{cc}
\operatorname{diag}\{L\} & \mathbf{0} \\
\mathbf{0} & C
\end{array}\right] \frac{d}{d t}\left[\begin{array}{l}
i \\
v
\end{array}\right]=\left[\begin{array}{cc}
\mathbf{0} & -\mathbf{1}_{m} \\
\mathbf{1}_{m}^{\boldsymbol{\top}} & -1 / R
\end{array}\right]\left[\begin{array}{l}
i \\
v
\end{array}\right]
$$

$$
+\left[\begin{array}{c}
\operatorname{diag}\{E\} \\
\mathbf{0}_{m}^{\top}
\end{array}\right] d
$$

Fig. 2 gives graphical representation of the system dynamics and exhibits a natural feedback of voltage $v$ into dynamics of $i$ which is governed by input $d$. This diagram clearly shows that control of $v$ can only by achieved "indirectly" through total current $\sigma$, driven by duty cycle $d$.

\subsection{A running example}

Relevance of the proposed control framework will be assessed by way of two distinct configurations: (i) the real experimental benchmark described in Section 6 and (ii) an academic example used throughout this paper to support didactic discussion. Characteristics of this example are as follows:

- The interconnection is made of $m=3$ converters whose electrical components are as follows

$$
\begin{gathered}
L_{1}=300 \mathrm{mH}, L_{2}=L_{3}=200 \mathrm{mH}, \\
E_{1}=E_{2}=24 \mathrm{~V}, E_{3}=20 \mathrm{~V} .
\end{gathered}
$$




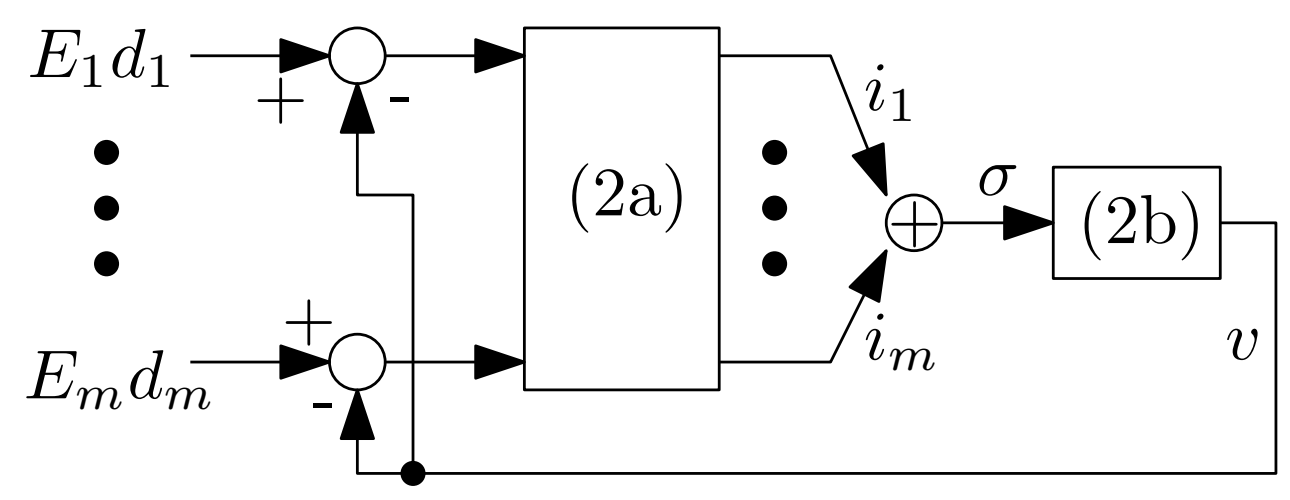

Figure 2: Open-loop model.

- For clarity of the exposition, simple balanced current-sharing is considered as control objective associated with this example, so that $x_{r}(R)=\left(\mathbf{1}_{m} v_{r} /(m R), v_{r}\right)$ and voltage reference $v_{r}$ is set to $12 \mathrm{~V}$

- Extreme values of the load is such that $\mathbb{L}=[0.1,1] \Omega$.

\section{Literature review}

This section outlines existing solutions of Problem 1. It focuses on "active current-sharing mechanisms" rather than (open-loop) conventional droop methods, since the latter does not achieve exact current and voltage regulation thus, does not appropriately answer to Problem $1[16,17]{ }^{3}$

Literature in this area is well surveyed in $[2,13]$. Remarkably, almost all existing solutions make use of a two nested loops scheme. This section first provides on overview of main implementations of this strategy. Then, its intrinsic limitation associated with the bandwidth of the two loops is discussed and illustrated.

\subsection{The ubiquitous two nested loops scheme}

As noticed in [2], control solution of Problem 1 are typically designed via a two-step procedure. The first step aims associating a close control to each converter making them individually acts as a either a controlled voltage source or a controlled current source. The second step seeks coordination of individual subsystems in order to achieve exact voltage and current regulation. The resulting control scheme adopts the two nested loops scheme depicted on Fig. 3. The inner loop results from first step procedure whereas the second step is concerned by outer loop design.

Rephrasing in terms of control theory, the first step aims designing a control law $\left(i_{k}, v\right) \mapsto d_{k}$ using local signal and such that the whole interconnected system admits an attractive equilibrium characterized by individual steady-state behavior of the form $v=v_{\text {ref }, k}-\alpha_{k} i_{k}$ for voltage-mode control and $i_{k}=$

\footnotetext{
${ }^{3}$ Saying it differently, conventional droop control can only drive closed-loop system toward a (load-dependent) neighborhood of $x_{r}(R)$ instead of this set-point itself.
} 


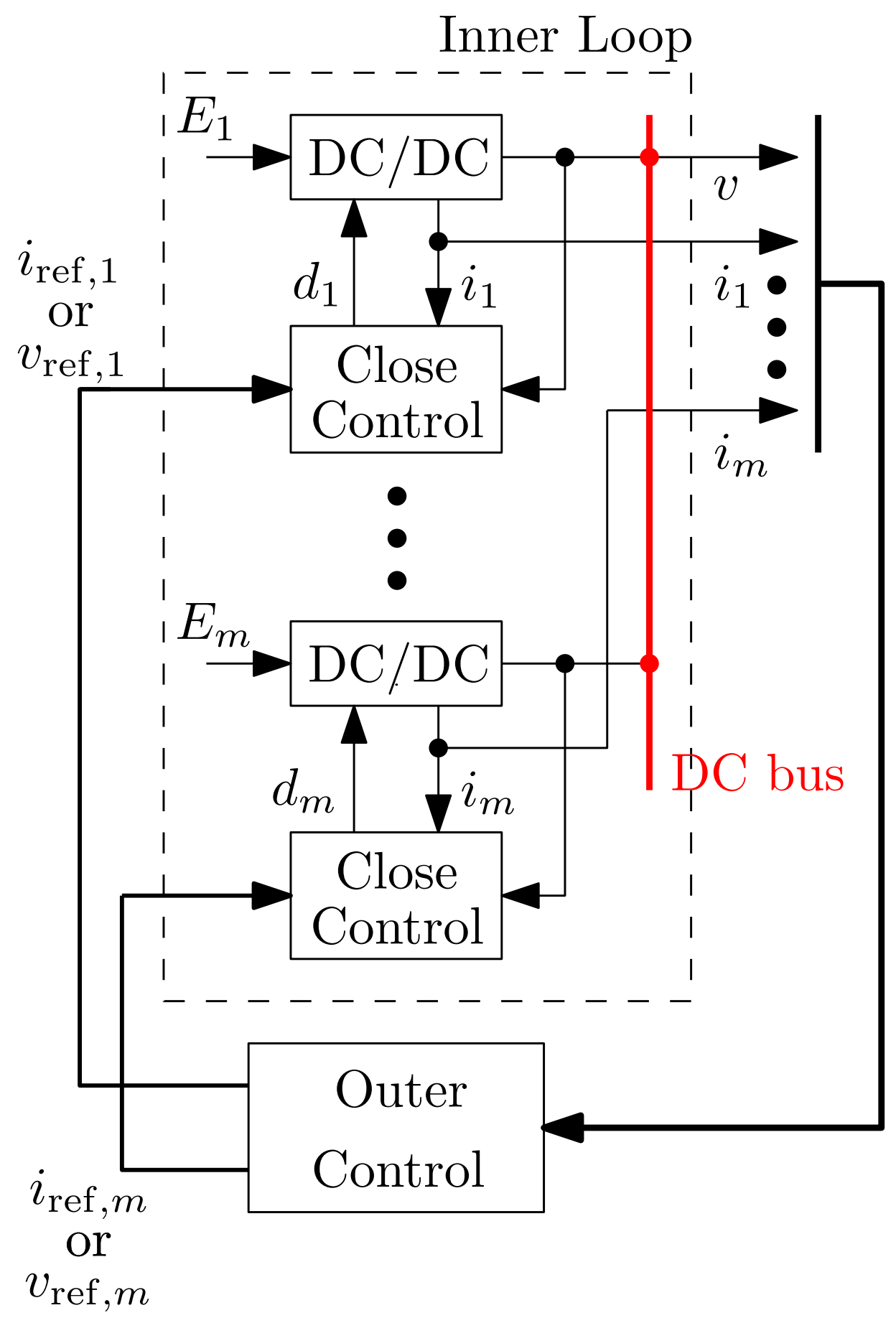

Figure 3: The two nested loops control scheme 
$i_{\text {ref }, k}-v / \beta_{k}$ for current-mode control. Signals $v_{\text {ref }, k}$ and $i_{\text {ref, } k}$ are exogenous signal (from the close control point of view) whereas $\alpha_{k}$ and $\beta_{k}$ are constant parameters depending on close controller gains as well as converter parameters and having units of $\Omega$. Relying on circuit theory considerations, it is shown in [2] that first step can only results in three different configurations: Parallel interconnection of imperfect voltage sources (type I), parallel interconnection of current sources plus one voltage source (type II) and parallel interconnection of current sources (type III). Note that theoretical proof of existence of some attractive equilibrium for the overall interconnection is seldom provided, even when $v_{\mathrm{ref}, k}$ and $i_{\mathrm{ref}, k}$ are assumed to be constant. Notable exception of this fact can be found in [14, 1], where complex impedances and transfer functions analysis is provided, and in [8], where state-space analysis via Lyapunov functional is performed.

When it comes to the second step, the goal is to design a high level control layer driving the whole system toward current and voltage reference by means of signals $v_{\text {ref }, k}$ and $i_{\text {ref, }, k}$. Usually, not only voltage $v$ but also current $i_{k}$ of each branches are fed back to this outer controller, in order to regulate voltage deviation $v_{r}-v$ as well as current deviation $i_{r}-i$ to zero, whatever is the load. Depending on the type of implemented inner loop, different outer control scheme can be used: For type I (resp. type III), the outer controller delivers voltage (current) references $v_{\text {ref, } k}\left(i_{\text {ref, }, k}\right)$ to each branch based on individual current and voltage deviation; For type II, the unique voltage source is responsible for voltage regulation and its own current serves as reference for other branches which are assimilated to current sources. Note that so-called "master/slave" and "democratic" strategies correspond to outer loops counter-part of type II and type III close control loops, respectively $[2,1]$.

\subsection{A simple type III control scheme implementation}

Let us illustrate general strategy outlined in previous subsection in the particular case of type III inner loop. This particular control structure has been indeed recognized as the most promising, due to fast transient, see [2, p.1103] and [13, p.907].

Close control loop is selected as a PI controller fed by current deviation with respect to $i_{\text {ref, } k}$ plus output voltage cancelling out natural feedback of $v$ into current dynamics (see Fig. 2):

$$
d_{k}(s)=\frac{K_{i, k}}{E_{k}} \frac{1+s / \omega_{i, k}}{s}\left(i_{\mathrm{ref}, k}(s)-i_{k}(s)\right)+\frac{v}{E_{k}} .
$$

Suitable gain selection imposes individual converters to behave as current controlled sources, i.e. if $i_{\text {ref,k}}(t)$ is a sufficiently slowly time-varying signal, then differences between this reference and $i_{k}(t)$ is small enough to be neglected so that $i_{k}(t)$ can be driven almost perfectly by way of $i_{\text {ref, } k}(t)$. At low frequency, resulting interconnection can be approximated via the following algebraic relationship

$$
v(s)=R \sum_{k \in \mathcal{K}} i_{\mathrm{ref}, k}(s)
$$

which is nothing but Kirchhoff's junction rule.

Let us borrow outer loop proposed in [2, Section V] for this type of inner loop and in the case of balanced current-sharing. Structure of this high level controller is depicted by Fig. 5 where transfer 
functions $H_{1}(s)$ and $H_{2, k}(s)$ read as follows:

$$
H_{1}(s)=\frac{K_{1}}{\epsilon} \frac{1+s / \omega_{1}}{s}, \quad H_{2, k}(s)=\frac{K_{2, k} / \epsilon}{1+s / \omega_{2, k}} .
$$

with $K_{1}=-4.8 \times 10^{4}, \omega_{1}=6 \times 10^{3}, K_{2, k}=-0.7$ and $\omega_{2, k}=1 \times 10^{5}$ for all $k$. Parameter $\epsilon$ is set to zero for the time being. Infinite DC gain of $H_{1}$ cancels steady-state voltage deviation and current deviation can be made negligible by increasing $K_{2, k}$.

Example. Let us perform simulations for the three bucks example introduced in Subsection 2.5. Controller parameters are selected as follows:

$$
\begin{aligned}
K_{i, k} & =\left[\begin{array}{lll}
11.84 & 7.90 & 7.90
\end{array}\right] \times 10^{5}, \\
\omega_{i, k} & =\left[\begin{array}{lll}
4.49 & 4.49 & 4.49
\end{array}\right] \times 10^{4}
\end{aligned}
$$

For initial conditions corresponding to $v(0)=0 \mathrm{~V}$ and $i(0)=[0,10,20] \mathrm{A}$, simulation results are depicted by Fig. 4 when $R=1 \Omega$ : Voltage regulation is asymptotically exact and all currents seemingly reaches the same value at the steady-state.

By modifying gains $1 / m$ (see Fig. 5), this control scheme can be easily adapt to deal with nonuniform current distribution if fixed current ratio is considered, i.e. each converter delivers a fixed percentage of the total current. Yet, going further by requiring this ratio to be load depend as in Problem 1, appears to be much more involved.

\subsection{Frequency separation as a fundamental tool for achieving closed-loop stability}

If its simplicity makes the two loops control solution attractive, particular care should be taken in its implementation. The nested loops may indeed badly interact. If this phenomena has already been reported in the literature (see e.g. $[2,13]$ ), theoretical stability certificate are seldom established. Indeed, in order to theoretically validate the approach, one has to taken variability of the load into account as well as bandwidth of each loops.

In order to highlight this phenomena, gains of transfer functions $H_{1}$ and $H_{2, k}$ have been parametrized by strictly positive real $\epsilon$ : The smaller is $\epsilon$, the higher is the gain of the transfer function and the larger is the bandwidth of the outer controller. Starting from $\epsilon=1$, leading to a satisfactory closed-loop system (see Fig. 4), this bandwidth is progressively enlarged, by decreasing $\epsilon$, up to reach instability. Extreme value of load domain $\mathbb{L}$ is considered and same initial conditions as for Fig. 4 are used. Chronograph of voltage trajectories are reported in Fig. 6: If either $\epsilon=1$ or $\epsilon=0.8$ lead to satisfactory results, instability is experienced for $\epsilon=0.6$ regardless of the value of $R$. This behavior is confirmed by location of poles of the closed-loop transfer function from $v_{r}$ to $v$ : As shown by Fig. 7 which focuses on $R=1 \Omega$, by decreasing $\epsilon$ from 1 to 0.6 , we move poles from pentagram to square, so that system becomes instable since one square belongs to the right hand side of the complex plan.

The above conclusion suggests that classical two loops control solution suffers from fundamental limitation: If satisfactory steady-state is achievable, transient performances are implicitly bounded. 

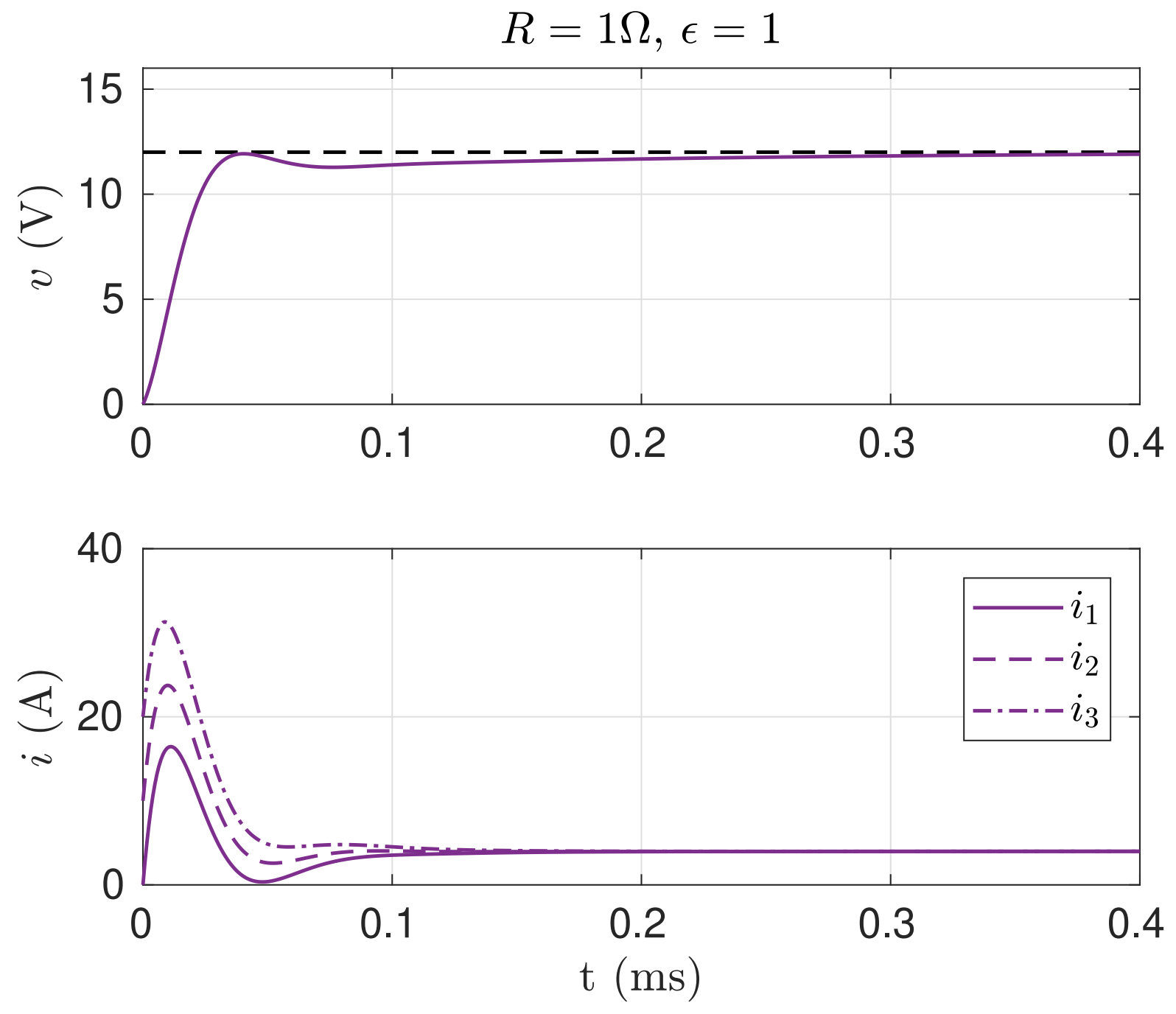

Figure 4: Step response of closed-loop system with two loops controller. 


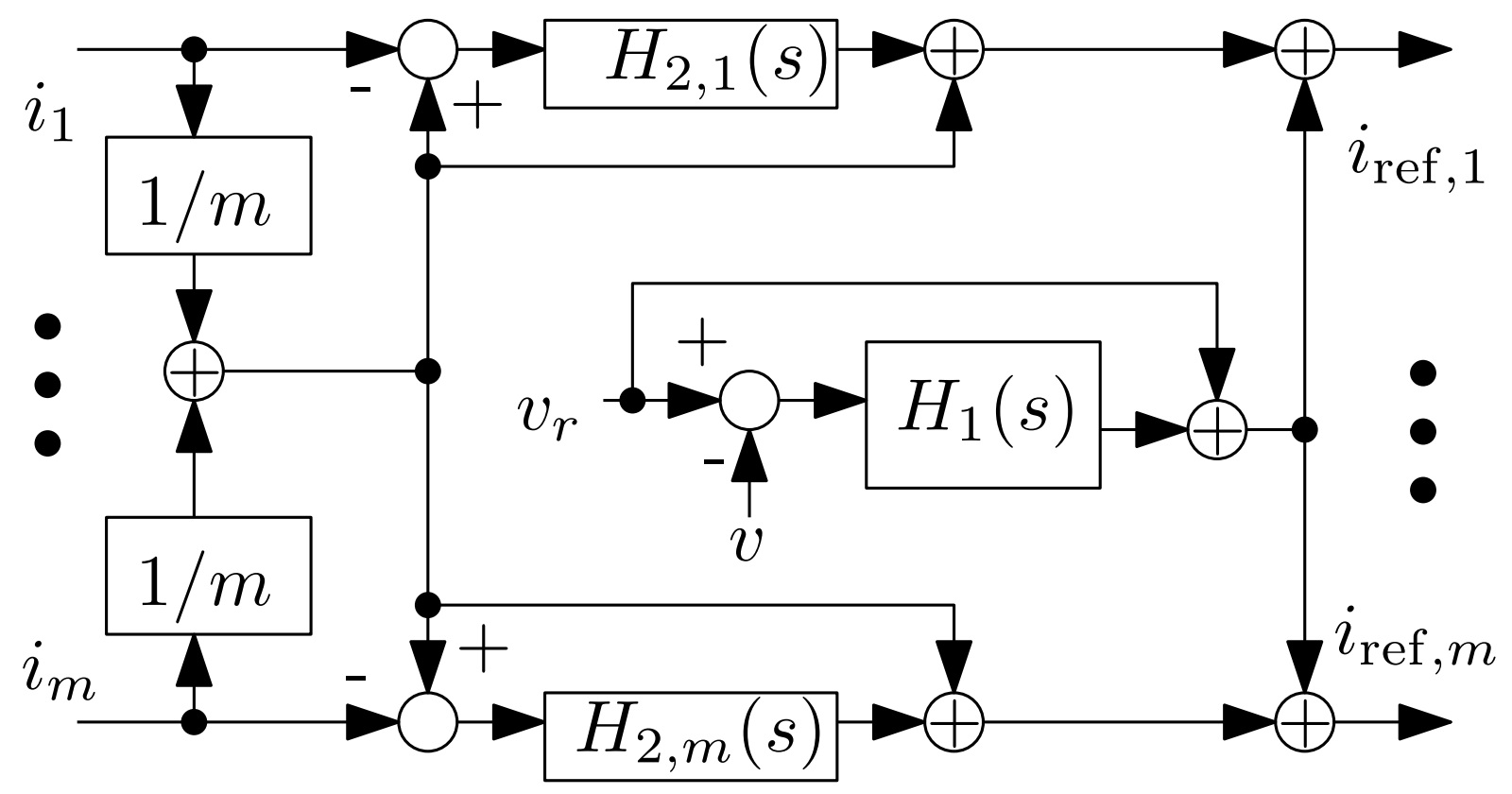

Figure 5: Outer loop for type III inner control scheme [2].
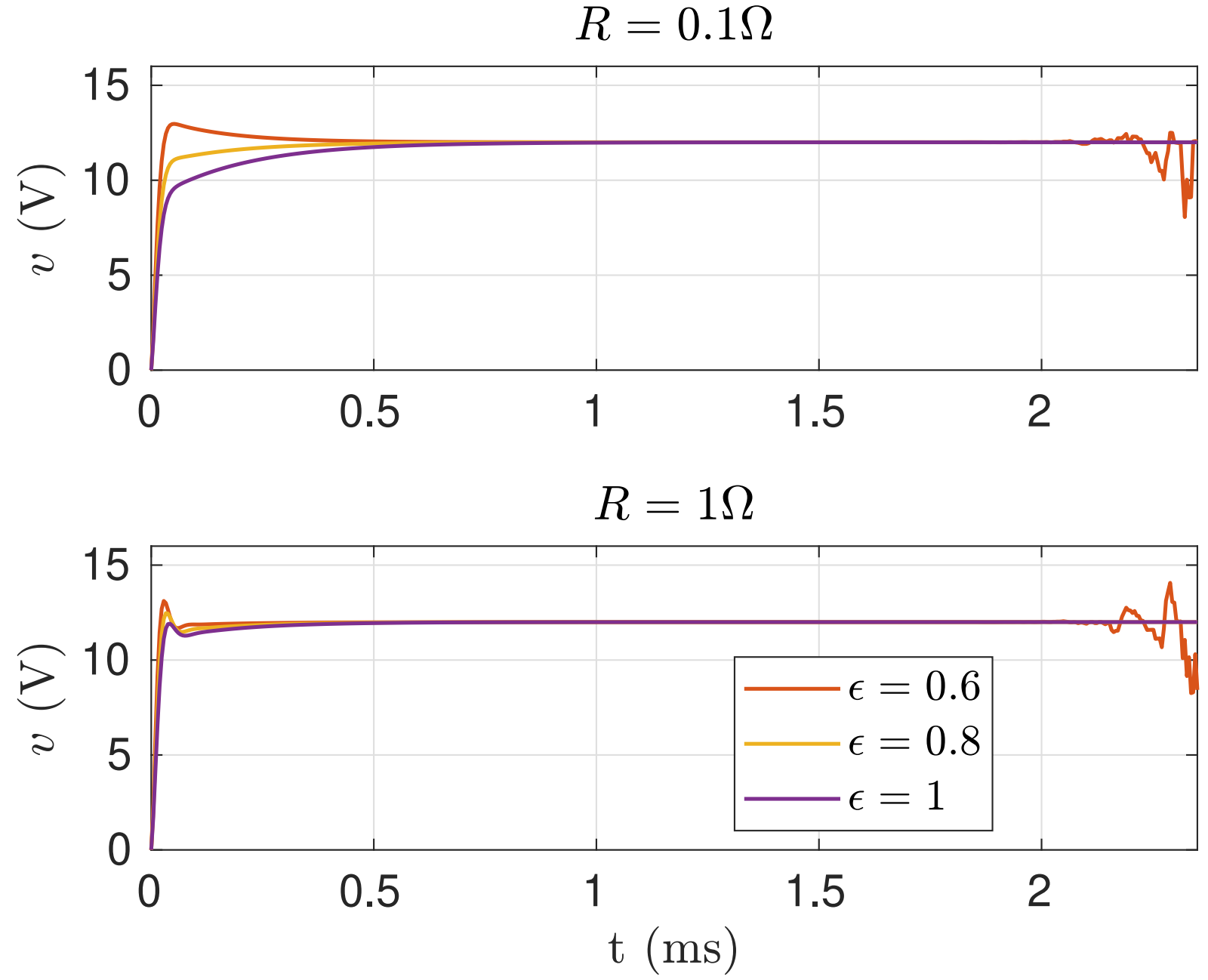

Figure 6: Impact of load value and gain of the outer loop on step response of closed-loop system with two loops controller. 


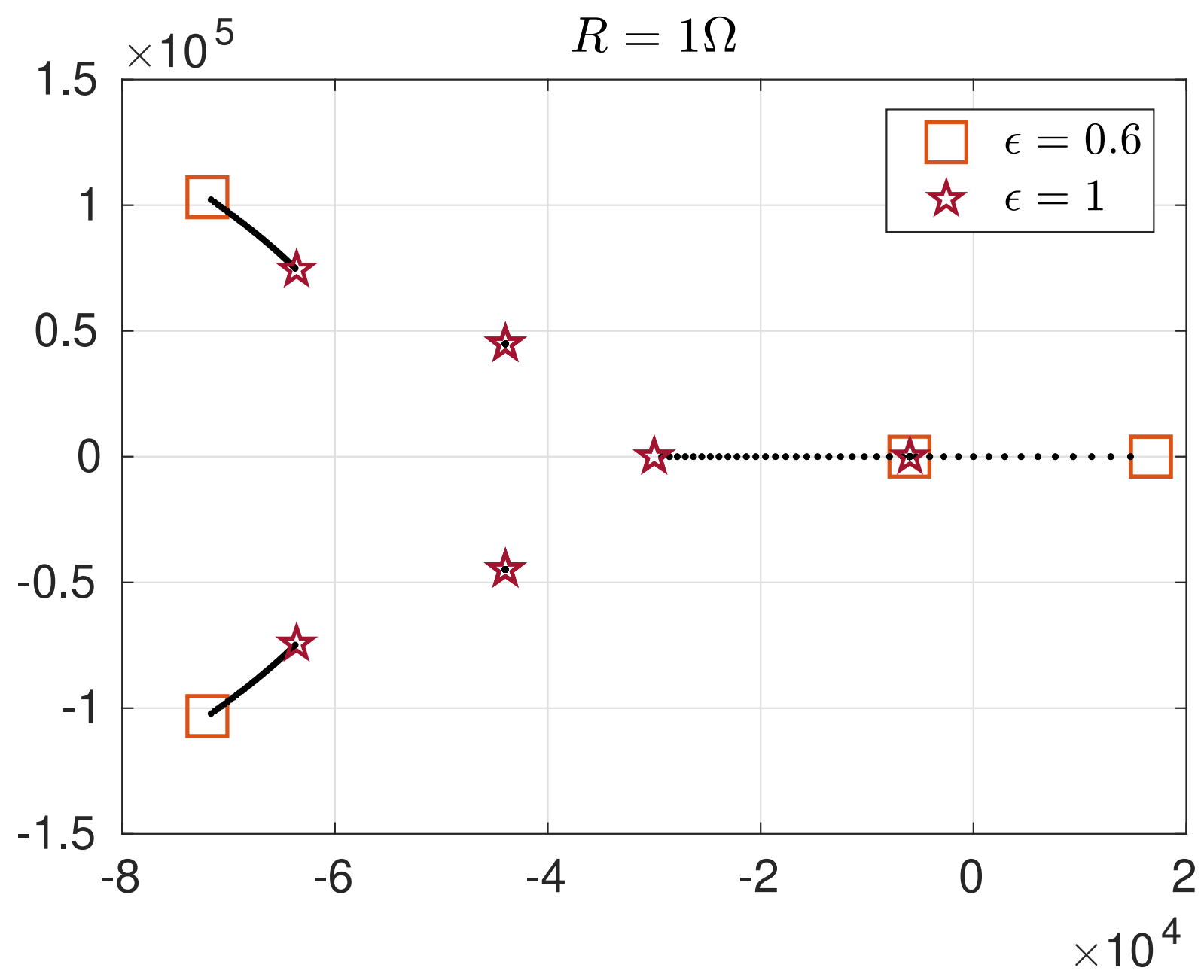

Figure 7: Motion of poles of the closed-loop transfer function from $v_{r}$ to $v$ when increasing gain of outer loop. 
Indeed, when trying to accelerate either voltage regulation or current-sharing dynamics, one ends up to increase gain of the outer loop so that frequency separation collapses and the two loops start interacting what, in turn, leads to instability.

\section{Geometrical decomposition of the dynamical model}

This section presents the main result of this paper. Reformulation of open-loop model (2) is first obtained before being interpreted from circuit theory view-point. Then, discussions about relevance of this result for control purpose ends the section. In stark contrast with the two nested loops scheme, our approach isolates current-sharing from voltage regulation (and vice versa) via geometric considerations and without resorting to frequency separation argument. It is shown that this strategy allows to outperform fundamental limitations of existing two loops controllers.

\subsection{A new basis}

As exhibited by (2b), voltage dynamics does not depend on each current $i_{k}$ individually but rather on total current $\sigma$. In order to better highlight this dependency, let us introduce the new coordinates $(\delta, \sigma, v) \in \mathbb{R}^{m-1} \times \mathbb{R} \times \mathbb{R}$ where $\sigma$ explicitly appears:

$$
\left[\begin{array}{l}
\delta \\
\sigma \\
v
\end{array}\right]=T^{-1}\left[\begin{array}{l}
i \\
v
\end{array}\right],
$$

with

$$
T^{-1}:=\left[\begin{array}{cc}
\Gamma_{m}^{\top} & \mathbf{0}_{m-1} \\
\mathbf{1}_{m}^{\top} & 0 \\
\mathbf{0}_{m}^{\top} & 1
\end{array}\right] \in \mathbb{R}^{(m+1) \times(m+1)},
$$

and where $\Gamma_{m} \in \mathbb{R}^{m \times(m-1)}$ is defined as follows

$$
\Gamma_{m}:=\left[\begin{array}{cccc}
1 & 0 & \cdots & 0 \\
-1 & 1 & \ddots & \vdots \\
0 & -1 & \ddots & 0 \\
\vdots & \ddots & \ddots & 1 \\
0 & \cdots & 0 & -1
\end{array}\right]=\left[\begin{array}{l}
\mathbf{I}_{m-1} \\
\mathbf{0}_{m-1}^{\top}
\end{array}\right]-\left[\begin{array}{l}
\mathbf{0}_{m-1}^{\top} \\
\mathbf{I}_{m-1}
\end{array}\right]
$$

Note that $T$ is indeed invertible as $\Gamma_{m}$ is a matrix basis of the orthogonal complement of $\operatorname{Im}\left\{\mathbf{1}_{m}\right\}$ in $\mathbb{R}^{m}$. It can be easily verified that $T$ reads

$$
T=\left[\begin{array}{ccc}
\Gamma_{m}\left(\Gamma_{m}^{\boldsymbol{\top}} \Gamma_{m}\right)^{-1} & \frac{1}{m} \mathbf{1}_{m} & \mathbf{0}_{m} \\
\mathbf{0}_{m-1}^{\boldsymbol{\top}} & 0 & 1
\end{array}\right] .
$$

It is worth mentioning that the new coordinate $\delta \in \mathbb{R}^{m-1}$ admits a simple physical interpretation: From (5), this signal is related to $i$ in the following way

$$
\delta=\Gamma_{m}^{\top} i=\left[\begin{array}{llll}
\left(i_{1}-i_{2}\right) & \left(i_{2}-i_{3}\right) & \cdots & \left(i_{m-1}-i_{m}\right)
\end{array}\right]^{\top},
$$


which shows that $\delta$ reflects current distribution. This is nothing but the component of $i$ which is missing in $\sigma$, as expected from invertibility of $T$.

In the rest of the paper, we shall use the operator $\Delta$ defined as follows

$$
\mathbb{R}^{p} \ni y \mapsto \Delta y:=\Gamma_{p}^{\top} y
$$

$$
=\left[\left(y_{1}-y_{2}\right) \cdots\left(y_{p-1}-y_{p}\right)\right]^{\top} \in \mathbb{R}^{p-1}
$$

so that $\delta$ could alternatively be written as $\Delta i$.

In this new basis, matrix equation (3) becomes

$$
\begin{array}{r}
\frac{d}{d t}\left[\begin{array}{c}
\delta \\
\sigma \\
v
\end{array}\right]=\left[\begin{array}{c|cc}
\mathbf{0} & \mathbf{0} & -\Gamma_{m}^{\top} \operatorname{diag}\{L\}^{-1} \mathbf{1}_{m} \\
\hline \mathbf{0} & 0 & -\mathbf{1}_{m}^{\top} \operatorname{diag}\{L\}^{-1} \mathbf{1}_{m} \\
\mathbf{0} & 1 / C & -1 /(R C)
\end{array}\right]\left[\begin{array}{c}
\delta \\
\frac{\sigma}{v}
\end{array}\right] \\
+\left[\begin{array}{c}
\frac{\Gamma_{m}^{\top} \operatorname{diag}\{L\}^{-1} \operatorname{diag}\{E\}}{\mathbf{1}_{m}^{\top} \operatorname{diag}\{L\}^{-1} \operatorname{diag}\{E\}} \\
\mathbf{0}_{m}^{\top}
\end{array}\right] d,
\end{array}
$$

since it holds

$$
\begin{aligned}
T^{-1} & {\left[\begin{array}{cc}
\operatorname{diag}\{L\} & \mathbf{0}_{m} \\
\mathbf{0}_{m}^{\top} & C
\end{array}\right]^{-1}\left[\begin{array}{cc}
\mathbf{0}_{m \times m} & -\mathbf{1}_{m} \\
\mathbf{1}_{m}^{\top} & -1 / R
\end{array}\right] T } \\
& =\left[\begin{array}{cc}
\Gamma_{m}^{\top} & \mathbf{0}_{m-1} \\
\mathbf{1}_{m}^{\top} & 0 \\
\mathbf{0}_{m}^{\top} & 1
\end{array}\right]\left[\begin{array}{ccc}
\mathbf{0} & \mathbf{0}_{m} & -\operatorname{diag}\{L\}^{-1} \mathbf{1}_{m} \\
\mathbf{0}_{m-1}^{\top} 1 / C & -1 /(R C)
\end{array}\right] \\
& =\left[\begin{array}{ccc}
\mathbf{0} & \mathbf{0} & -\Gamma_{m}^{\top} \operatorname{diag}\{L\}^{-1} \mathbf{1}_{m} \\
\mathbf{0} & 0 & -\mathbf{1}_{m}^{\top} \operatorname{diag}\{L\}^{-1} \mathbf{1}_{m} \\
\mathbf{0} & 1 / C & -1 /(R C)
\end{array}\right]
\end{aligned}
$$

and ASM. 1 can be written as

$$
\begin{aligned}
& {\left[\begin{array}{lll}
\delta_{r}^{\top}(R) & \sigma_{r}(R) & v_{r}
\end{array}\right]^{\top}:=T^{-1}\left[\begin{array}{ll}
i_{r}^{\top}(R) & v_{r}
\end{array}\right]^{\top}} \\
& \in \operatorname{Ker}\left\{\left[\begin{array}{ll}
R \mathbf{1}_{m}^{\top} & -1
\end{array}\right] T\right\} \\
& =\operatorname{Ker}\left\{\left[\begin{array}{lll}
\mathbf{0}_{m-1}^{\top} & R & -1
\end{array}\right]\right\} \text {, }
\end{aligned}
$$

for all $R \in \mathbb{L}$. Note that this set membership is nothing but the equilibrium condition of voltage dynamics (2b), i.e.:

$$
\sigma_{r}(R)=v_{r} / R
$$

Example. For the three bucks example introduced in Subsection 2.5, new state vector reads as follows:

$$
\left(\delta_{1}, \delta_{2}, \sigma, v\right)=\left(i_{1}-i_{2}, i_{2}-i_{3}, i_{1}+i_{2}+i_{3}, v\right)
$$


Balanced current-sharing objective is considered so that entries of current reference vector are identical, i.e. $i_{r}(R)=v_{r} /(m R) \mathbf{1}_{m}$. In such a case, current distribution reference $\delta_{r}=\left(i_{r, 1}-i_{r, 2}, i_{r, 2}-i_{r, 3}\right)$ is identically null and, hence, does not depend on $R$ unlike $\sigma_{r}=i_{r, 1}+i_{r, 2}+i_{r, 3}=v_{r} / R$.

\subsection{Block decomposition}

State matrix (10) admits a block triangular structure which allows to interpret the system as a cascade of two dynamical blocs: An upper-subsystem, governing coupled dynamics of $(\sigma, v)$, feeds a lower-one, describing $\delta$ dynamics, with $v$. This cascaded structure originates from the independence of dynamics of $\delta$ from $(\sigma, v)$, so that the upper-subsystem impacts the lower-one but there is no signal in the other way around.

Input $d$ acts on both subsystems. To better understand this coupling, let us proceed to the change of input coordinates

$$
\begin{aligned}
d=\operatorname{diag}\{E\}^{-1} \operatorname{diag}\{L\}\left[\Gamma_{m}\left(\Gamma_{m}^{\mathbf{T}} \Gamma_{m}\right)^{-1}\right. & \left.\frac{1}{m} \mathbf{1}_{m}\right] \\
& {\left[\begin{array}{cc}
\operatorname{diag}\left\{\Delta^{\star}\left(L^{-1}\right)\right\} \operatorname{diag}\left\{\Delta^{\star} E\right\} & \mathbf{0} \\
\mathbf{0} & E_{\text {eq }} / L_{\text {eq }}
\end{array}\right]\left[\begin{array}{l}
\lambda \\
\mu
\end{array}\right] }
\end{aligned}
$$

which decomposes $d$ into $\lambda \in \mathbb{R}^{m-1}$ and $\mu \in \mathbb{R}$. In this relationship, the following constants have been introduced:

$$
\begin{aligned}
\mathbb{R} \ni 1 / L_{\mathrm{eq}} & :=\mathbf{1}_{m}^{\top} \operatorname{diag}\{L\}^{-1} \mathbf{1}_{m}=\sum_{k} 1 / L_{k} \\
\mathbb{R} \ni E_{\mathrm{eq}} & :=\min _{k} E_{k} .
\end{aligned}
$$

The operator $\Delta^{\star}$ derives from $\Delta$, defined by (9), as follows

$$
\mathbb{R}^{p} \ni y \mapsto\left(\Delta^{\star} y\right)_{k}:= \begin{cases}(\Delta y)_{k}, & \left(y_{k} \neq y_{k+1}\right) \\ 1, & \text { (otherwise) }\end{cases}
$$

and, with slight abuse of notation, the inverse of any vector $y \in \mathbb{R}^{p}$ for which $y_{k} \neq 0,(k \in\{1, \ldots, p\})$ corresponds to component-wise inversion, i.e.

$$
y^{-1}:=\operatorname{diag}\{y\}^{-1} \mathbf{1}_{p}=\left[\begin{array}{lll}
1 / y_{1} & \cdots & 1 / y_{p}
\end{array}\right]^{\top}
$$

Note that the use of $\Delta^{\star}$ instead of $\Delta$ in (11) ensures that $d \mapsto(\lambda, \mu)$ is a bijection as $\operatorname{diag}\left\{\Delta^{\star}\left(L^{-1}\right)\right\} \operatorname{diag}\left\{\Delta^{\star} E\right\}$

is always invertible. ${ }^{4}$

\footnotetext{
${ }^{4}$ Obviously, this assertion holds provided that the problem is physically meaningful in the sense that both $E_{k}$ and $L_{k}$ are non-zero for all $k \in \mathcal{K}$.
} 


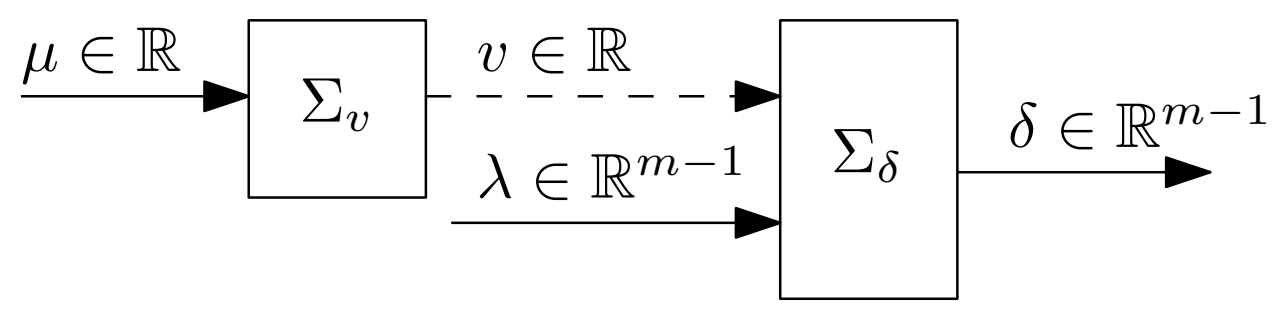

Figure 8: New cascaded open-loop model.

The resulting dynamics of the open-loop system reduces to

$$
\frac{d}{d t}\left[\begin{array}{c}
\delta \\
\hline \sigma \\
v
\end{array}\right]=\left[\begin{array}{c|cc}
\mathbf{0} & \mathbf{0} & -\Delta\left(L^{-1}\right) \\
\hline \mathbf{0} & 0 & -1 / L_{\mathrm{eq}} \\
\mathbf{0} & 1 / C & -1 /(R C)
\end{array}\right]\left[\begin{array}{c}
\delta \\
\hline \sigma \\
v
\end{array}\right]
$$

$$
+\left[\begin{array}{cc}
\operatorname{diag}\left\{\Delta^{\star}\left(L^{-1}\right)\right\} \operatorname{diag}\left\{\Delta^{\star} E\right\} & \mathbf{0} \\
\hline \mathbf{0} & E_{\mathrm{eq}} / L_{\mathrm{eq}} \\
\mathbf{0} & 0
\end{array}\right]\left[\begin{array}{l}
\lambda \\
\mu
\end{array}\right],
$$

so that the upper-subsystem $\Sigma_{v}$ governs voltage dynamics through total current $\sigma$ controlled by input $\mu$

$$
\Sigma_{v}: \frac{d}{d t}\left[\begin{array}{l}
\sigma \\
v
\end{array}\right]=\left[\begin{array}{cc}
0 & -1 / L_{\mathrm{eq}} \\
1 / C & -1 /(R C)
\end{array}\right]\left[\begin{array}{l}
\sigma \\
v
\end{array}\right]+\left[\begin{array}{c}
E_{\mathrm{eq}} / L_{\mathrm{eq}} \\
0
\end{array}\right] \mu,
$$

and drives the lower-subsystem $\Sigma_{\delta}$ corresponding to dynamics of $\delta$ driven by control signal $\lambda$

$$
\begin{aligned}
\Sigma_{\delta}: \frac{d}{d t} \delta=-\Delta\left(L^{-1}\right) v & \\
& +\operatorname{diag}\left\{\Delta^{\star}\left(L^{-1}\right)\right\} \operatorname{diag}\left\{\Delta^{\star} E\right\} \lambda
\end{aligned}
$$

or, equivalently,

$$
\Sigma_{\delta}^{k}:\left\{\begin{aligned}
(\Delta L)_{k} \frac{d}{d t} \delta_{k} & =-v+\left(\Delta^{\star} E\right)_{k} \lambda_{k}, & \left(L_{k} \neq L_{k+1}\right) \\
\frac{d}{d t} \delta_{k} & =\left(\Delta^{\star} E\right)_{k} \lambda_{k}, & \text { (otherwise), }
\end{aligned}\right.
$$

for all $k \in\{1, \ldots, m-1\}$. The last relationship has been obtained by left-multiplying (15) by $\operatorname{diag}\left\{\Delta^{\star}\left(L^{-1}\right)\right\}^{-1}$.

Fig. 8 illustrates this structure.

Example. Consider example introduced in Subsection 2.5 and for which $L_{1} \neq L_{2}=L_{3}$ and $E_{1}=E_{2} \neq E_{3}$.

In this case, it holds

$$
\begin{aligned}
\Delta^{\star}\left(L^{-1}\right) & =\left[\begin{array}{ll}
\left(1 / L_{1}-1 / L_{2}\right) & 1
\end{array}\right]^{\top}, \\
\Delta^{\star} E & =\left[\begin{array}{ll}
1 & \left(E_{2}-E_{3}\right)
\end{array}\right]^{\top} .
\end{aligned}
$$

Subsystem $\Sigma_{\delta}$ reads

$$
\begin{aligned}
\left(L_{1}-L_{2}\right) \dot{\delta}_{1} & =-v+\lambda_{1} \\
\dot{\delta}_{2} & =\left(E_{2}-E_{3}\right) \lambda_{2}
\end{aligned}
$$


and (11) gives

$$
\begin{aligned}
& \frac{E_{1}}{L_{1}} d_{1}-\frac{E_{2}}{L_{2}} d_{2}=\left(\frac{1}{L_{1}}-\frac{1}{L_{2}}\right) \lambda_{1} \\
& \frac{E_{2}}{L_{2}} d_{2}-\frac{E_{3}}{L_{3}} d_{3}=\left(E_{2}-E_{3}\right) \lambda_{2} \\
& \frac{E_{1}}{L_{1}} d_{1}+\frac{E_{2}}{L_{2}} d_{2}+\frac{E_{3}}{L_{3}} d_{3}=\left(\min _{k} E_{k}\right)\left(\sum_{k} \frac{1}{L_{k}}\right) \mu
\end{aligned}
$$

so that $\lambda_{1,2}$ are related to differences of duty cycles (and drives $\delta=\Delta i$ ), whereas $\mu$ reflects the sum $d_{1}+d_{2}+d_{3}$ by which $\sigma=i_{1}+i_{2}+i_{3}$ is controlled.

1) From previous subsection, it comes out that $\lambda$ parametrizes the part of $d$ which is invisible by $\sigma$ and hence $v$, whereas $\mu$ is the remaining part by which $\sigma$ can be affected. As a result, regulation of $v$ boils down to the design of the single input controller

$$
\mathcal{C}_{v}: \quad(\sigma, v) \mapsto \mu,
$$

which is actually nothing but voltage regulation problem for a single buck converter.

2 ) The new formulation allows for modular design. Indeed, both voltage $v$ (via $\sigma$ ) and current distribution $\delta$ can be controlled independently by way of $\mu$ and $\lambda$, respectively. If this assertion clearly holds for $\Sigma_{v}$, effect of $v$ on $\Sigma_{\delta}$ can also be canceled out even when $L_{k} \neq L_{k+1}$ by simply substituting $\lambda$ by $\lambda^{\prime}+v /\left(\Delta^{\star} E\right)_{k}$ via an inner feedback loop. In such a case, one ends up with two disconnected subsystems, as suggested by the dashed line of Fig. 8 . 


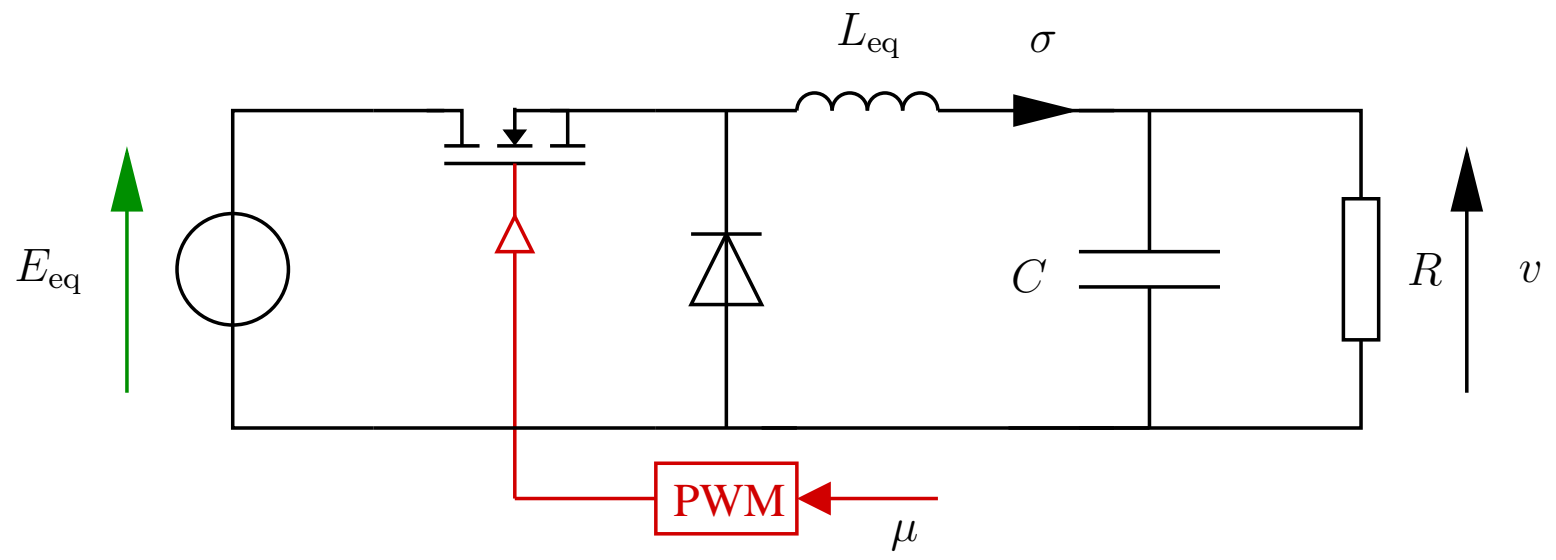

(a)
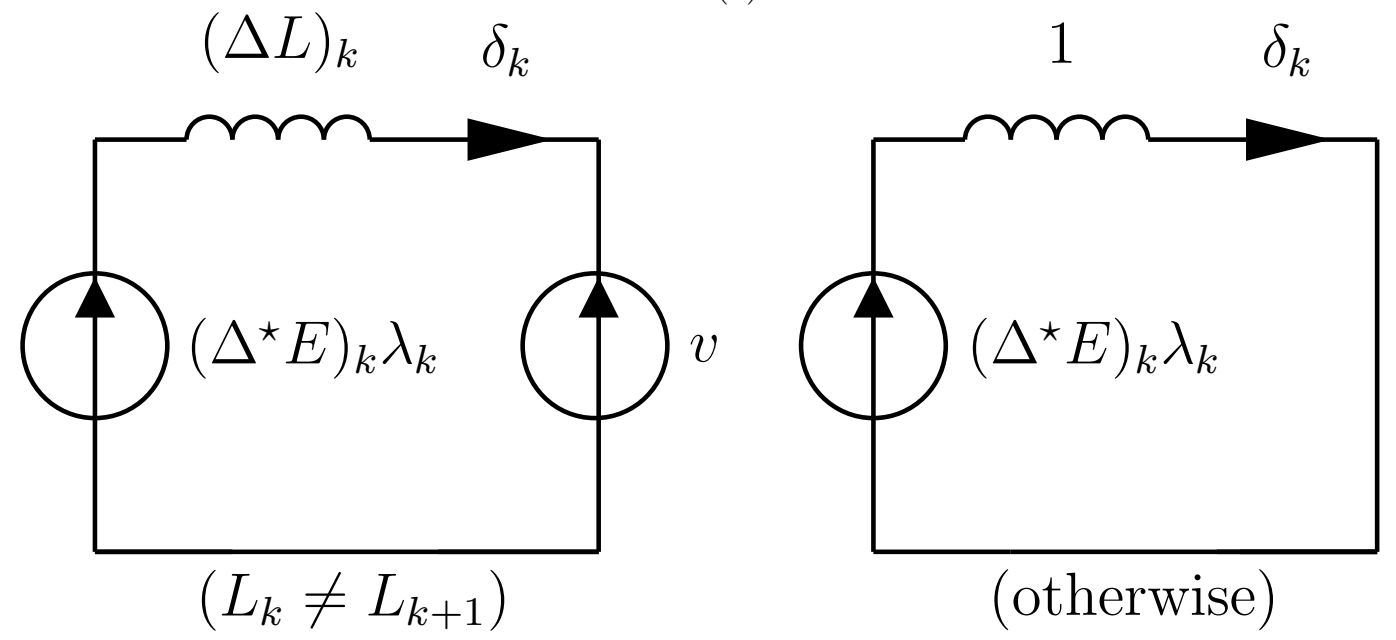

(b)

Figure 9: Circuit theory interpretation of (a) $\Sigma_{v}$ and (b) $\Sigma_{\delta}^{k}$ in the two cases. 


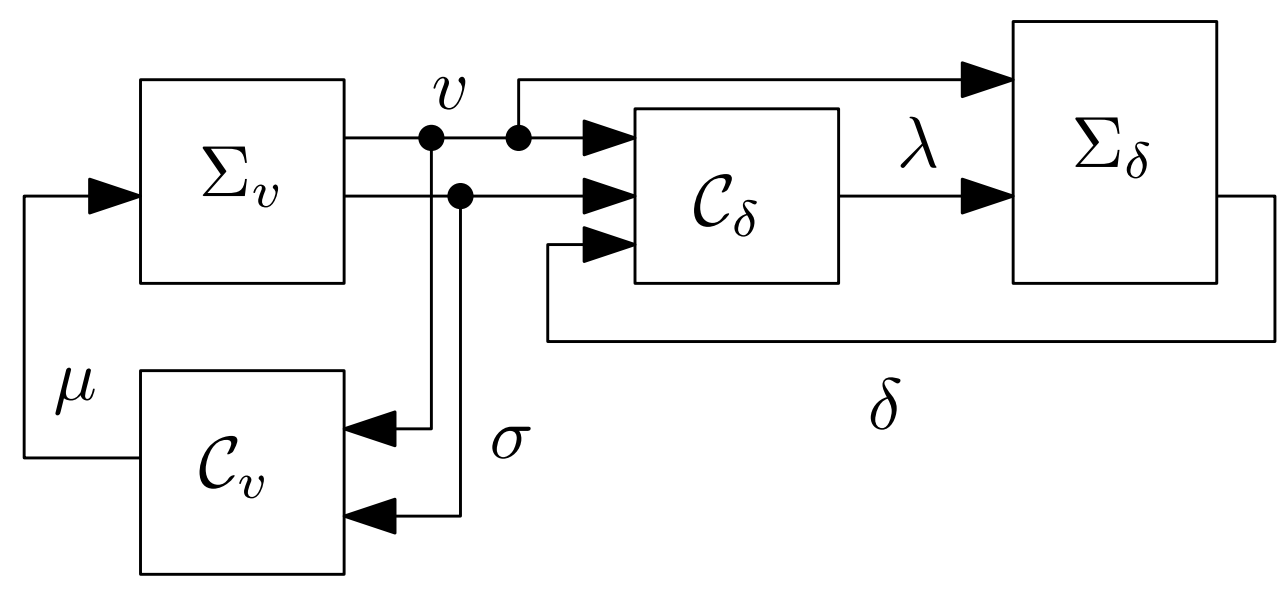

Figure 10: Proposed control scheme.

Nevertheless, apart from the special case where $\delta_{r}(\cdot)$ is constant, it should be notice that load have to be estimated by controller of $\Sigma_{\delta}$ in order to properly define the desired reference $\delta_{r}(R)$. As a result, some information have to be convey from $\Sigma_{v}$ to $\Sigma_{\delta}$ for this estimation to be possible since $\Sigma_{\delta}$ does not depend on $R$. For this reason, the map

$$
\mathcal{C}_{\delta}: \quad(\delta, \sigma, v) \mapsto \lambda,
$$

is considered as the general form of controller for $\Sigma_{\delta}$.

3) Ordering of the cascade preserves hierarchy of control objectives. Indeed, in general, lower subsystem of cascaded system converges after the upper one has reached its equilibrium. As a result, leading control goals have preferably to be fulfill by highest subsystem. The proposed control scheme complies with this guideline as voltage regulation, performed by $\Sigma_{v}$, dominates power-flow control, related to $\Sigma_{\delta}$, in the control objective hierarchy.

Still, $v$ cannot be controlled directly by control input, but only through $\sigma$. However, open-loop inertia of the total current $\sigma$ is related to $L_{\text {eq }}$ which is typically very small, as parallel interconnection of coils ends up with a reduced equivalent inductance.

\section{Control design for unknown load}

The purpose of this section is to illustrate the relevance of the proposed control structure depicted by Fig. 10. A procedure leading to subcontrollers $\mathcal{C}_{v}$ and $\mathcal{C}_{\delta}$ is provided together with theoretical certificate that the overall resulting controller solves Problem 1, see Theorem 5.3. This procedure is expected to be sufficiently simple to fulfill didactic purpose, yet leaving large room for improvement. Example introduced in Subsection 2.5 will be used as a didactical illustration.

\subsection{Control of individual subsystems}

Exploiting cascaded structure depicted by Fig. 10, proof of stability of

$$
(\delta, \sigma, v)=\left(\delta_{r}(R), v_{r} / R, v_{r}\right)
$$


(see subsection 4.1) can be established via classical tools for such an interconnection. This allows to

cope with non-linearities which might be introduced by load estimation (see subsection 5.1.2). In this context, stability for the overall system can be inferred from stability of $(\sigma, v)=\left(v_{r} / R, v_{r}\right)$ for $\mathcal{C}_{v} \star \Sigma_{v}$ and 0-stability of $\delta=\delta_{r}(R)$ for $\mathcal{C}_{\delta} \star \Sigma_{\delta}$ (that is stability of $\mathcal{C}_{\delta} \star \Sigma_{\delta}$ when $\mathcal{C}_{v} \star \Sigma_{v}$ is at the steady-state), as well as boundedness of trajectories [18]. ${ }^{5}$

For this reason, stability for individual closed-loop subsystems $\mathcal{C}_{v} \star \Sigma_{v}$ and $\mathcal{C}_{\delta} \star \Sigma_{\delta}$ are first obtained and then used as main ingredients to ensure stability for the whole system.

\subsubsection{Control of $\Sigma_{v}$ (voltage regulation)}

As $\Sigma_{v}$ can be interpreted as a virtual buck converter, every technique which aims regulating this system are applicable, see e.g. [19, 20]. In particular, let us use state-feedback with output integrator of the form $-k_{p}\left(v_{r}-v\right)-k_{d} \sigma-k_{i} / C \int\left(v_{r}-v\right)$, so that $\mathcal{C}_{v}$ adopts the following formulation ${ }^{6}$

$$
\mathcal{C}_{v}:\left\{\begin{aligned}
\dot{z} & =\left(v_{r}-v\right) / C \\
\mu & =-k_{i} z-k_{p}\left(v_{r}-v\right)-k_{d} \sigma .
\end{aligned}\right.
$$

Next lemma gives sufficient condition for controller gains computation.

Lemma 5.1. Let load dependent matrix $A(R)$ be defined as

$$
\mathbb{R}^{3 \times 3} \ni A(R):=\left[\begin{array}{ccc}
0 & -1 / L_{\mathrm{eq}} & 0 \\
1 / C & -1 /(R C) & 0 \\
0 & -1 / C & 0
\end{array}\right] .
$$

If there exist $W=W^{\top} \in \mathbb{R}^{3 \times 3}$ and $Y \in \mathbb{R}^{1 \times 3}$ such that $W \succ 0$ and

$$
\begin{array}{r}
W A^{\top}(R)+A(R) W+\left[\begin{array}{lll}
E_{\text {eq }} / L_{\text {eq }} & 0 & 0
\end{array}\right]^{\top} Y+ \\
Y^{\top}\left[\begin{array}{lll}
E_{\text {eq }} / L_{\text {eq }} & 0 & 0
\end{array}\right] \prec 0
\end{array}
$$

holds for all $R \in \partial \mathbb{L}=\{\underline{R}, \bar{R}\}$, then gains computed via

$$
\left[\begin{array}{ccc}
-k_{d} & k_{p} & -k_{i}
\end{array}\right]=Y W^{-1}
$$

ensure that $(\tilde{\sigma}, \tilde{v})=\mathbf{0}$ is a globally exponentially stable (GES) equilibrium for closed-loop (14) with (16) and for all $R \in \mathbb{L}$.

Proof. Stability of the equilibrium satisfying $v=v_{r}$ is achieved if dynamics of $\mathcal{C}_{\delta} \star \Sigma_{\delta}$, described by the

\footnotetext{
${ }^{5}$ Here, notation $\mathcal{P}_{1} \star \mathcal{P}_{2}$ refers to the interconnection of $\mathcal{P}_{1}$ and $\mathcal{P}_{2}$.

${ }^{6}$ Here, integral gain $k_{i}$ is scaled via $C$ in order to improve conditionning of matrices involved in the design of controller parameters.
} 
following equation, is stable for all $R \in \mathbb{L}$ :

$$
\frac{d}{d t}\left[\begin{array}{l}
\sigma \\
v \\
z
\end{array}\right]=\underbrace{\left(A(R)+\left[\begin{array}{c}
E_{\text {eq }} / L_{\text {eq }} \\
0 \\
0
\end{array}\right]\left[\begin{array}{c}
-k_{d} \\
k_{p} \\
-k_{i}
\end{array}\right]^{\top}\right)}_{=: \hat{A}(R)}\left[\begin{array}{l}
\sigma \\
v \\
z
\end{array}\right]
$$

$$
+\left[\begin{array}{c}
-E_{\mathrm{eq}} / L_{\mathrm{eq}} k_{p} \\
0 \\
1 / C
\end{array}\right] v_{r}
$$

This condition is achieved if (17) is satisfied for all $R \in \partial \mathbb{L}$. Indeed, in such a case, condition (17) holds for all $R \in \mathbb{L}$ by convexity argument. Then, substituing $Y$ by $\left[\begin{array}{lll}-k_{d} & k_{p} & -k_{i}\end{array}\right] W$ gives $W \hat{A}^{\top}(R)+\hat{A}(R) W \prec$ 0 which, in turn, proves quadratic stability of closed-loop dynamics [21].

Example. Design of $\mathcal{C}_{v}$ can be alternatively tackled via a transfer function strategy. In this case, the goal is to find a proper rational function $k(s)$ such that poles of $k(s) f_{\mu \rightarrow v}(R ; s) /\left(1+k(s) f_{\mu \rightarrow v}(R ; s)\right)$ lie in the open left half complex plane, for all $R \in \mathbb{L}$. Here, load-dependent transfer function $f_{\mu \rightarrow v}(R ; s)$ characterized dynamical relationship between $\mu$ and $v$ and reads (see (14)):

$$
f_{\mu \rightarrow v}(R ; s)=\frac{E_{\mathrm{eq}} R}{R L_{\mathrm{eq}} C s^{2}+L_{\mathrm{eq}} s+R}
$$

By means of Routh-Hurwitz stability criterion, one can be easily checked that PI controller

$$
k(s)=k_{c}\left(1+s / \omega_{c}\right) / s
$$

solves the problem if $k_{c}>\left(\underline{R} C E_{\text {eq }}\right)^{-1}$ and $\omega_{c}<\left(\bar{R} C-1 /\left(k_{c} E_{\text {eq }}\right)\right)^{-1}$.

\subsubsection{Control of $\Sigma_{\delta}$ (current distribution assignment)}

Controller $\mathcal{C}_{\delta}$ has to ensure 0 -stability of $\delta=\delta_{r}(R)$, that is stability when $\Sigma_{v}$ has reached its equilibrium $(\sigma, v)=\left(v_{r} / R, v_{r}\right)$. Since current distribution $\delta_{r}$ to be achieved is load dependent, it is necessary to estimate $R$. Remarking that equilibrium points in the state-space must verify $v=R \sigma$, computation of $v_{r} / \sigma$ can serve as a simple load-estimator which asymptotically converges to $R$, provided that $v$ goes to $v_{r}$ as $t$ tends to infinity: In such a case, $\sigma(t)$ must converges to $v_{r} / R$ so that $v_{r} / \sigma(t)$ tends to $R$.

Next lemma shows that the following static map is a suitable candidate

$$
\mathcal{C}_{\delta}: \quad \lambda=\operatorname{diag}\left\{\Delta^{\star} E\right\}^{-1} \operatorname{diag}\left\{\Delta^{\star}\left(L^{-1}\right)\right\}^{-1}
$$

$$
\left(-K\left(\delta_{r}(\hat{R}(\sigma))-\delta\right)+\Delta\left(L^{-1}\right) v_{r}\right)
$$

whenever $K$ is Hurwitz, and where $\hat{R}: \mathbb{R} \rightarrow \mathbb{L}=[\underline{R}, \bar{R}]$ reads

$$
\hat{R}(\sigma):= \begin{cases}\bar{R}, & \left(\sigma<v_{r} / \bar{R}\right) \\ v_{r} / \sigma, & \left(v_{r} / \bar{R} \leq \sigma \leq v_{r} / \underline{R}\right) \\ \underline{R}, & \left(\sigma>v_{r} / \underline{R}\right) .\end{cases}
$$

and is depicted by Fig. 11 . 


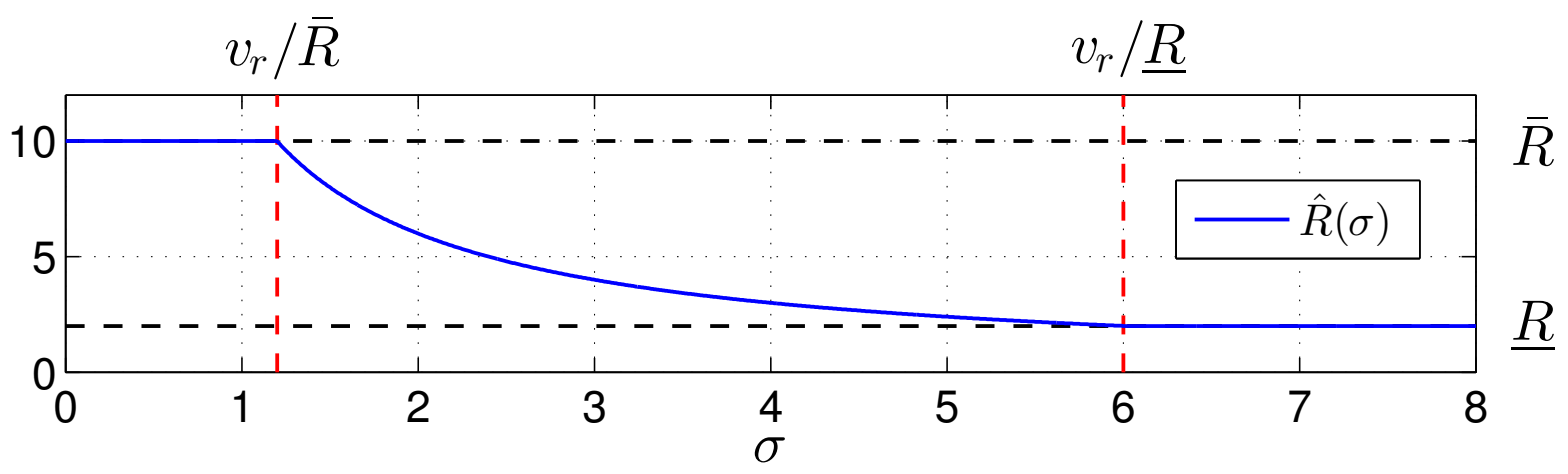

Figure 11: Load estimator $\hat{R}(\sigma)$.

Remark (Definition of $\hat{R}$ ). Load estimation via $v_{r} / \sigma$ introduces non-linearity in the controller $\mathcal{C}_{\delta}$. Yet, cascaded structure of the closed-loop can be exploited to cope with this difficulty, as demonstrated by next subsection. Also, special care have to be taken to ensure that $\delta_{r}\left(v_{r} / \sigma\right)$ remains bounded. As shown by Fig. 11, this issue is addressed by saturating the quantity $v_{r} / \sigma$ outside $\mathbb{L}$ and, in turn, prevent division by zero. Since $x_{r}(R)$ has been assumed to be reachable, image of $\mathbb{L}$ by $\delta_{r}$ is bounded so that $\delta_{r}\left(v_{r} / \sigma\right)$ as well.

Lemma 5.2. If $(\sigma, v)=\left(v_{r} / R, v_{r}\right)$, then $\delta=\delta_{r}(R)$ is a GES equilibrium of closed-loop (15) and (19) for all Hurwitz matrix $K \in \mathbb{R}^{(m-1) \times(m-1)}$ and for all $R \in \mathbb{L}$.

Proof. When $(\sigma, v)=\left(v_{r} / R, v_{r}\right)$ and $R \in \mathbb{L}$, closed-loop of (15) and (19) reads $\dot{\delta}=K\left(\delta-\delta_{r}(R)\right)$ and, hence, $\delta$ asymptotically converges to $\delta_{r}(R)$ whenever $K$ is Hurwitz.

Example. Let us consider example of Subsection 2.5. In this case, controller $\mathcal{C}_{\delta}$ reads (recall that $\delta_{r} \equiv \mathbf{0}$ ):

$$
\lambda=\operatorname{diag}\left\{\frac{L_{1} L_{2}}{L_{2}-L_{1}}, \frac{1}{E_{2}-E_{1}}\right\}(K \delta
$$

$$
\left.+\left[\begin{array}{c}
1 / L_{2}-1 / L_{1} \\
0
\end{array}\right] v_{r}\right)
$$

Note that this expression reduces to

$$
\left[\begin{array}{l}
\lambda_{1} \\
\lambda_{2}
\end{array}\right]=\left[\begin{array}{c}
-v_{r}+\kappa \frac{L_{1} L_{2}}{L_{2}-L_{1}} \delta_{1} \\
\frac{\kappa}{E_{2}-E_{1}} \delta_{2}
\end{array}\right]
$$

by using simple choice $K=-\kappa \mathbf{I}_{2}$.

Remark (Independent dynamics). Dependency of dynamics of $\delta$ with respect to $v$ can be canceled out, in some particular case. Suppose that $\delta_{r}$ is constant (as for example of Subsection 2.5), then interconnection $\mathcal{C}_{\delta} \star \Sigma_{\delta}$ is governed by $\dot{\delta}=K\left(\delta-\delta_{r}\right)$ if $v$ is used in place of $v_{r}$ in (19). In such a case, $\mathcal{C}_{v} \star \Sigma_{v}$ and $\mathcal{C}_{\delta} \star \Sigma_{\delta}$ are fully decoupled so that Fig. 10 exhibits disconnected subsystems instead of unilateral relationship, i.e. $(\sigma, v)$ does not impact $\delta$ anymore. 


\subsection{Stability of the overall system}

Let us make the following technical assumption.

Assumption 2. Map $\delta_{r}(\cdot)$ is Lipschitz continuous, i.e. there exists $\beta \geq 0$ such that $\delta_{r}\left(R_{1}\right)-\delta_{r}\left(R_{2}\right) \leq$ $\beta\left(R_{1}-R_{2}\right)$ holds for all $\left(R_{1}, R_{2}\right) \in \mathbb{L}^{2}$.

Using this hypothesis and stability results of individual closed-loop subsystems $\mathcal{C}_{v} \star \Sigma_{v}$ and $\mathcal{C}_{\delta} \star \Sigma_{\delta}$, the following theorem demonstrates stability of $x=x_{r}(R)$ for the overall system.

Theorem 5.3. Under ASM. 1 and ASM. 2, the map $(i, v) \mapsto d$ described by (11), with $\mu$ delivered by (16) and $\lambda$ defined as in (19), solves Prob. 1 if $K$ is Hurwitz and $\left(k_{p}, k_{i}, k_{d}\right)$ are computed as in Lemma 5.1.

Proof. Let us define the following relative coordinates

$$
\tilde{x}:=\left[\begin{array}{cccc}
\tilde{\delta}^{\top} & \tilde{\sigma} & \tilde{v} & \tilde{z}
\end{array}\right]^{\top}:=\left[\begin{array}{llll}
\delta^{\top} & \sigma & v & z
\end{array}\right]^{\top}
$$

$$
-\left[\begin{array}{llll}
\delta_{r}^{\top}(R) & v_{r} / R & v_{r} & z^{\star}
\end{array}\right]^{\top}
$$

where $z^{\star}$ refers to the asymptotic value of $z$. If (i) $(\tilde{\sigma}, \tilde{v}, \tilde{z})=\mathbf{0}$ is a GAS equilibrium for $\mathcal{C}_{v} \star \Sigma_{v}$, (ii) $\tilde{\delta}=\mathbf{0}$ is a 0 -GAS equilibrium for $\mathcal{C}_{\delta} \star \Sigma_{\delta}$ (that is GAS when $(\tilde{\sigma}, \tilde{v}, \tilde{z})=\mathbf{0}$ ) and (iii) system trajectories are globally bounded, then $\tilde{x}=\mathbf{0}$ is a GAS equilibrium [18, Th.1.1]. Observe that properties (i) and (ii) have been established by Lemmas 5.1 and 5.2.

Let us now prove (iii). By global boundedness (GB) of the closed-loop system, we mean that for each $r>0$, there exists $\Psi(r)>0$ such that for each initial condition $\tilde{x}_{0}$ satisfying $\left|\tilde{x}_{0}\right| \leq r$, one has that all solutions $\tilde{x}$ satisfy $|\tilde{x}(t)| \leq \Psi(r)$ for all $t \geq 0$. To show this, first note that GAS of $\mathcal{C}_{v} \star \Sigma_{v}$ implies GB of the $(\tilde{\sigma}, \tilde{v}, \tilde{z})$ substate. Then, consider the function $H=|\tilde{\delta}|^{2} / 2$. From (15) and (19), we get:

$$
\begin{aligned}
\dot{H} & =\tilde{\delta}^{\top} \dot{\tilde{\delta}}^{\prime} \tilde{\delta}^{\top}\left(K\left(\delta-\delta_{r}(\hat{R}(\sigma))\right)-\Delta\left(L^{-1}\right) \tilde{v}\right) \\
& =\tilde{\delta}^{\top} \underbrace{\left(K\left(\delta_{r}(R)-\delta_{r}(\hat{R}(\sigma))\right)+\Delta\left(L^{-1}\right) \tilde{v}\right)}_{=: \rho(\sigma, \tilde{v})}+\tilde{\delta}^{\top} K \tilde{\delta} .
\end{aligned}
$$

Then remarking that $\tilde{\delta}^{\top} K \tilde{\delta}<0$ holds for all $\tilde{\delta}$ since $K$ is Hurwirtz, $\dot{H}$ can be bounded as follows:

$$
\dot{H} \leq|\tilde{\delta}||\rho| \leq\left(1+|\tilde{\delta}|^{2}\right)|\rho|=(1+2 H)|\rho| .
$$

As proved in the sequel, (a) there exist positive $k_{\rho}, \lambda_{\rho}, t_{0}$ such that $|\rho(t)| \leq k_{\rho} \exp \left(-\lambda_{\rho} t\right)$ for all $t \geq t_{0}$ and (b) $|\rho(t)|$ is bounded for $t \in\left[0, t_{0}\right]$. Then, from Gronwall-Bellman's inequality [22, Lemma A.1], since $\rho(t)$ is integrable, we have that $H=|\tilde{\delta}|^{2} / 2$ is globally bounded.

Let us now prove (a). First observe that $(\tilde{\sigma}, \tilde{v}, \tilde{z})$ dynamics is actually GES, so that for each $r>0$ there exist positive $k_{\sigma}, \lambda_{\sigma}, k_{v}, \lambda_{v}$ such that $|\tilde{\sigma}(t)| \leq k_{\sigma} \exp \left(-\lambda_{\sigma} t\right)$ and $|\tilde{v}(t)| \leq k_{v} \exp \left(-\lambda_{v} t\right)$. As a result, and from the fact that $R$ belongs to $\mathbb{L}$, there exists $t_{0} \geq 0$ such that inequalities

$$
v_{r}(\underbrace{1 / \bar{R}-1 / R}_{<0}) \leq \tilde{\sigma}(t) \leq v_{r}(\underbrace{1 / \underline{R}-1 / R}_{>0})
$$


hold for all $t \geq t_{0}$. By adding $\sigma_{r}=v_{r} / R$ on both side, those inequalities equivalently read $v_{r} / \bar{R} \leq \sigma(t) \leq$ $v_{r} / \underline{R}$ so that $\hat{R}(\sigma(t))=v_{r} / \sigma(t)$ holds, by definition of $\hat{R}$. This, in turn, proves that

$$
R-\hat{R}(\sigma(t))=v_{r}\left(\frac{1}{\sigma_{r}}-\frac{1}{\sigma(t)}\right)=v_{r} \frac{\sigma(t)-\sigma_{r}}{\sigma_{r} \sigma(t)}=\frac{R}{\sigma(t)} \tilde{\sigma}(t)
$$

and, hence,

$$
|R-\hat{R}(\sigma(t))| \leq \frac{R \bar{R}}{v_{r}}|\tilde{\sigma}(t)|
$$

are satisfied for all $t \geq t_{0}$. Using ASM. 2, it follows that

$$
\begin{aligned}
\left|\rho\left(\sigma, v-v_{r}\right)\right| \leq & |K|\left|\delta_{r}(R)-\delta_{r}(\hat{R}(\sigma))\right| \\
& +\left|\Delta\left(L^{-1}\right)\right|\left|v_{r}-v\right| \\
\leq & |K| \beta|R-\hat{R}(\sigma)|+\left|\Delta\left(L^{-1}\right)\right|\left|v_{r}-v\right| \\
\leq & |K| \beta \frac{R \bar{R}}{v_{r}} k_{\sigma} \exp \left(-\lambda_{\sigma} t\right) \\
& +\left|\Delta\left(L^{-1}\right)\right| k_{v} \exp \left(-\lambda_{v} t\right) \\
\leq & k_{\rho} \exp \left(-\lambda_{\rho} t\right)
\end{aligned}
$$

with

$$
\begin{aligned}
& k_{\rho}=\max \left\{|K| \beta \frac{R \bar{R}}{v_{r}} k_{\sigma},\left|\Delta\left(L^{-1}\right)\right| k_{v}\right\}>0 \\
& \lambda_{\rho}=\min \left\{\lambda_{\sigma}, \lambda_{v}\right\}>0
\end{aligned}
$$

This proves (a). In order to demonstrates (b), it suffices to invoke boundedness of $\mathbb{L}$, the image of $\hat{R}$, and the fact that $\tilde{v}(t)$ is bounded for all $t \geq 0$, so that

$$
\begin{aligned}
|\rho(\sigma, \tilde{v})| & \leq|K|\left|\delta_{r}(R)-\delta_{r}(\hat{R}(\sigma))\right|+\left|\Delta\left(L^{-1}\right)\right||\tilde{v}| \\
& \leq|K| M+\left|\Delta\left(L^{-1}\right)\right| k_{v}
\end{aligned}
$$

holds for all $t \leq t_{0}$, and where $M:=\max _{\left(R_{1}, R_{2}\right) \in \mathbb{L}^{2}} \delta_{r}\left(R_{1}\right)-\delta_{r}\left(R_{2}\right)$ is finite since ASM. 2 holds.

\subsection{Controller in the original coordinates}

As a conclusion of this design part, controller expression in the original coordinates is now provided, i.e. mapping $(i, v) \mapsto d$. It can be derived by using Fig. 12 and making use of change of coordinates (11) and (5) as well as subcontrollers expression $\mathcal{C}_{v}$ and $\mathcal{C}_{\delta}$ provided by (16) and (19), respectively.

Observe that complexity of the resulting controller dramatically decreases by selecting gain matrix of $\mathcal{C}_{\delta}$ as $K=-\kappa \mathbf{I}$. Indeed, in such a case, one gets (see Appendix A)

$$
d_{k}=\frac{L_{k}}{E_{k}}\left(\kappa\left(\frac{\sigma}{m}-i_{k}\right)+w_{k}(\sigma, \mu)\right),
$$

where $w_{k}$ is $k$-th entry of the following vector:

$$
\begin{aligned}
\mathbb{R}^{m} \ni w(\sigma, \mu)=\frac{1}{m} \mathbf{1}_{m} E_{\text {eq }} / L_{\text {eq }} \mu & \\
& +\Gamma_{m}\left(\Gamma_{m}^{\top} \Gamma_{m}\right)^{-1}\left(\kappa \delta_{r}(\hat{R}(\sigma))+\Delta\left(L^{-1}\right) v_{r}\right) .
\end{aligned}
$$




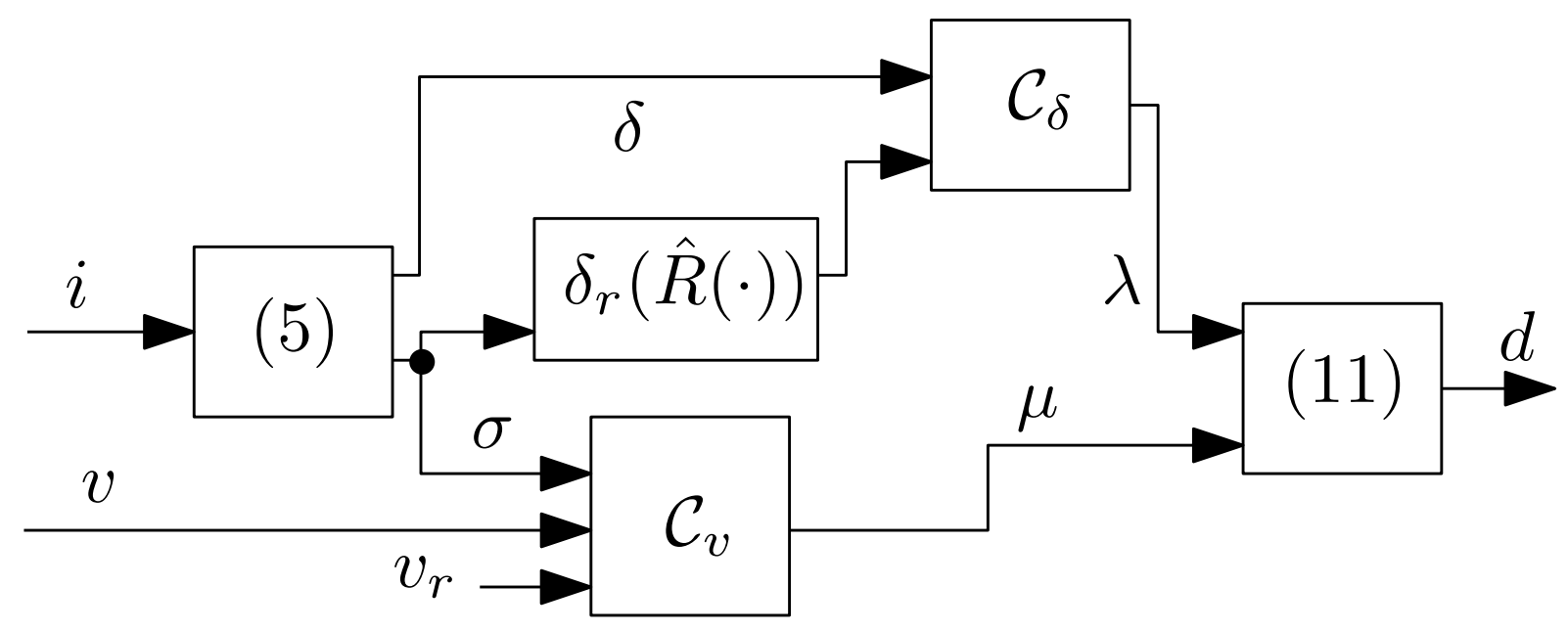

Figure 12: Proposed control scheme.

Thus, final controller is made of a proportional compensator on individual current $i_{k}$ whose gain is $\kappa$ and reference corresponds to average current $\sigma / m$. Output of this compensator is then shifted by $w_{k}(\sigma, \mu)$ which only depends on signals related to voltage dynamics, namely $\sigma$ and $\mu$.

Remark. Observe that when $\delta_{r}$ is constant, $w_{k}$ becomes independent on $\sigma$. In particular, whenever $\delta_{r} \equiv \mathbf{0}$ holds (as for example of Subsection 2.5), $w_{k}$ further reduces to

$$
w_{k}(\mu)=E_{\text {eq }} / L_{\text {eq }}(\mu / m)+b_{k} v_{r}
$$

where $k$-th entry of $\Gamma_{m}\left(\Gamma_{m}^{\top} \Gamma_{m}\right)^{-1} \Delta\left(L^{-1}\right)$ is denoted by $b_{k}$. Hence, $w_{k}(\mu)$ does not depend on $\sigma$ anymore and boils down to a linear combination of voltage reference $v_{r}$ and $\mu / m$, corresponding to a fixed portion of the duty cycle of virtual buck $\Sigma_{v}$.

Suppose further that subcontroller $\mathcal{C}_{v}$ is designed via a transfer function strategy (see example at the end of Subsection 5.1.1). In this case, one typically generates signal $\mu$ from voltage deviation $v_{r}-v$ via linear differential relationship captured by some transfer function $k(s)$. The resulting controller adopts the scheme depicted by Fig. 13, which ressembles to that of Fig. 5, i.e. outer controller of the classical two loops controller considered in Section 3.2. If the two schemes seemingly share common features, they differ mainly on the following aspects: (i) Signal of Fig. 5 which is alike to $w_{k}$ is identical for all converter whereas, on Fig. 13, $b_{k}$ makes computation of this signal dependent on $k$ and (ii) output signal of Fig. 13 is already duty cycle vector $d$ whereas controller of Fig. 13 produces $i_{\text {ref }}$, a mere internal signal of the overall controller. This discussion gives rise to an additional outcome of this paper: Even if controller of Fig. 13 has been elaborated following a non standard perspective, it can be regarded as some outer control law of the classical two loops controller considered in Section 3.2. Yet, controller of Fig. 13 benefits from the following features: (i) No inner loop is required (so that no frequency separation is required) and (iii) clear guidelines about how to select parameter of the controller are provided together with formal stability proof (see the forthcoming subsection). Let us emphasized that this controller should merely be regarded as a particular example of what broader framework proposed in this paper 


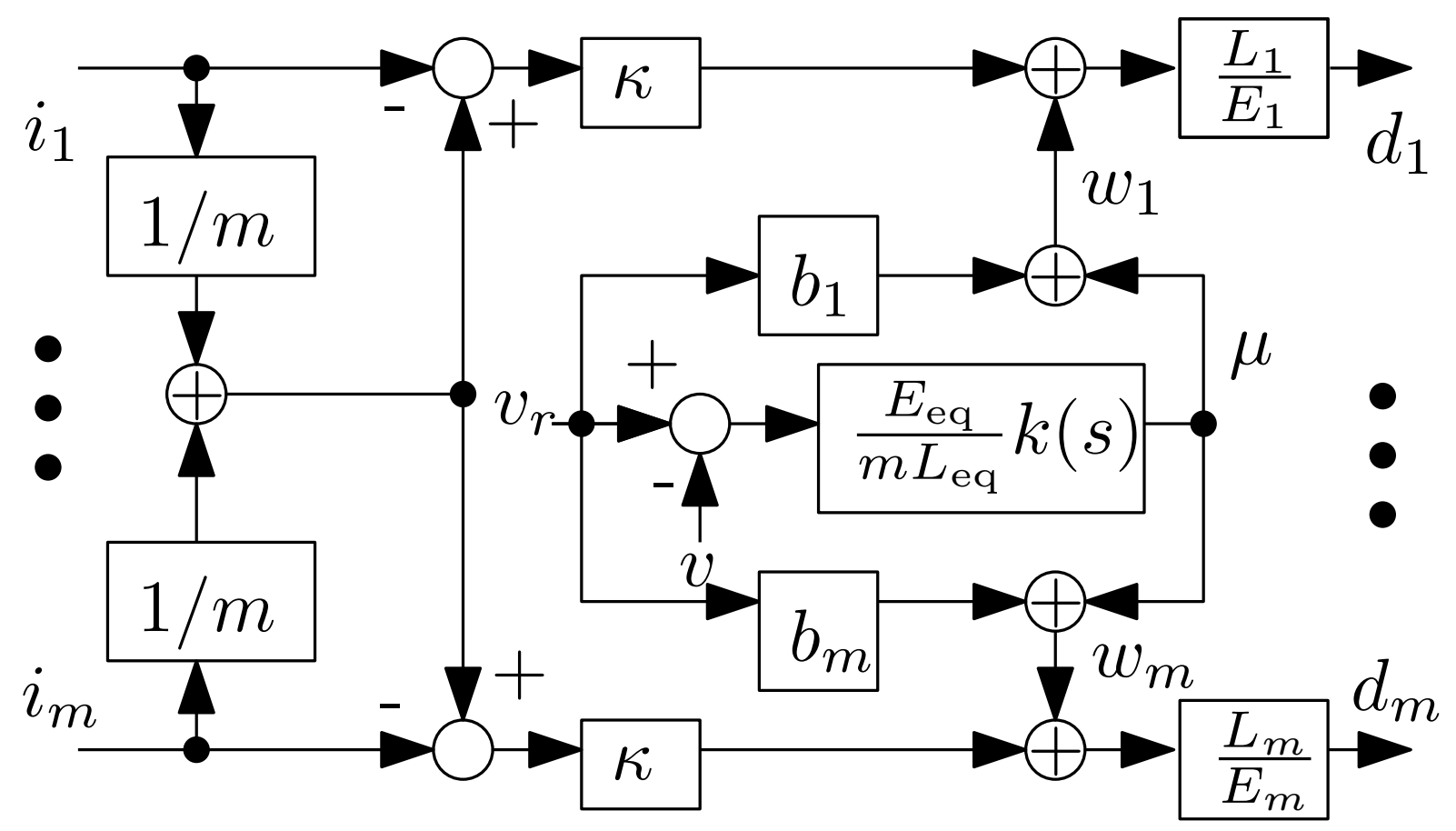

Figure 13: Proposed control scheme for $K=-\kappa \mathbf{I}$ and $\delta_{r} \equiv \mathbf{0}$.

\section{Minimizing steady-state power losses under power constraints}

In order to illustrate the proposed approach, let us consider the physically relevant scenario where closed-loop system has to asymptotically converges to the set-point minimizing overall power losses while achieving exact voltage regulation, i.e. $v(t)$ must converge to $v_{r}$. In addition to that, desired steadystate must comply with admissible power limits associated with each branch. This corresponds to select (load-dependent) set-point $x_{r}(R)$ via the following optimization problem

$$
\min _{i, v} J(i, v) \quad \text { s.t. } \quad v=v_{r},\left.\frac{d i_{k}}{d t}\right|_{k \in \mathcal{K}}=\frac{d v}{d t}=0, i \leq \bar{i}
$$

where $J$ gives total power losses and, according to [12, Eq. (11)], reads

$$
J(i)=\sum_{k \in \mathcal{K}} r_{1, k} i_{k}^{2}+r_{2, k} i_{k}
$$



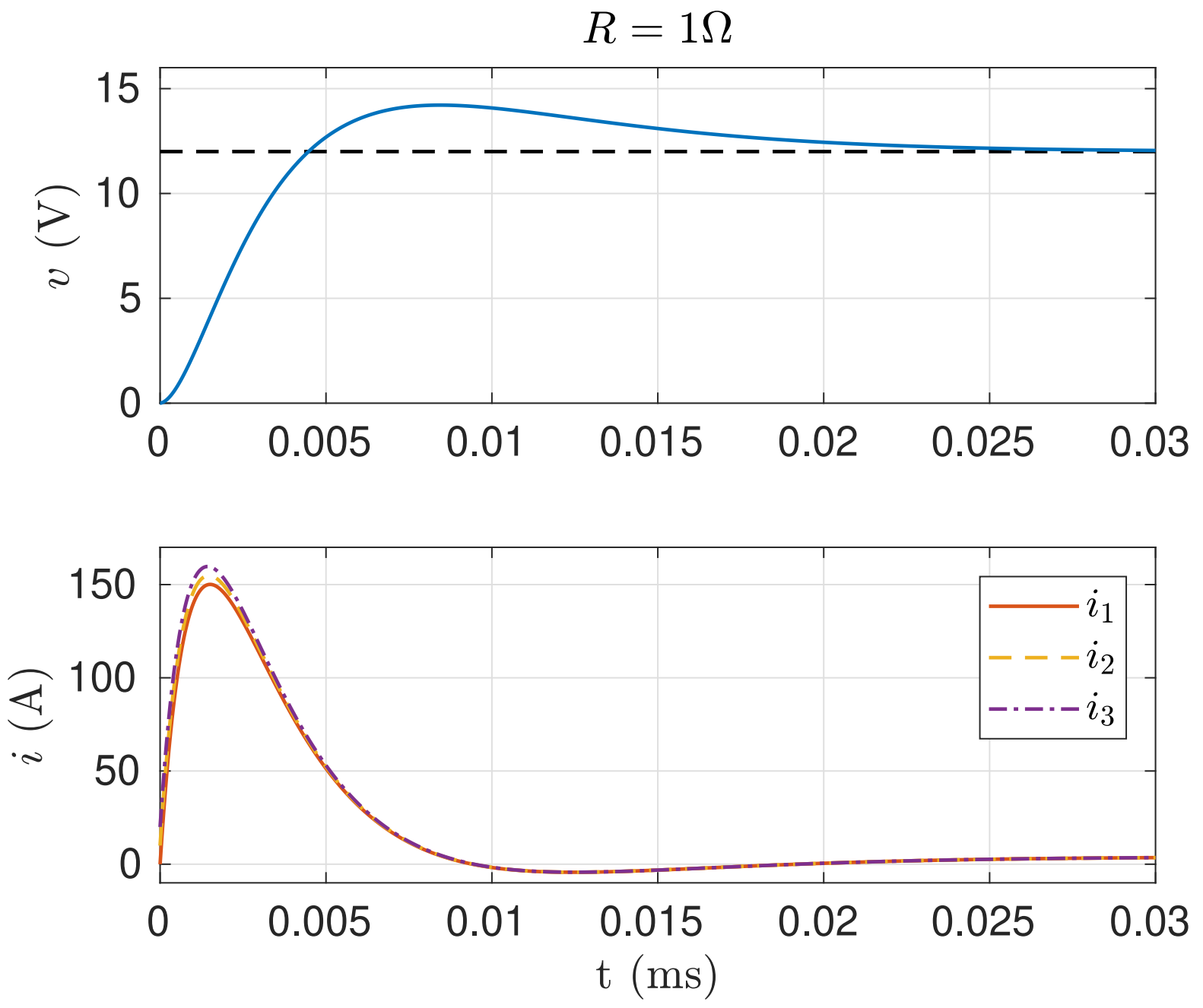

Figure 14: Closed-loop simulation results obtained via new controller (20) 
for some positive constants $r_{1, k}$ and $r_{2, k}$ depending on circuit parameters. As compared to (22), problem (22) incorporates inequality $i \leq \bar{i}$ in order to prevent each $i_{k}$ to exceed prescribed upper-bounds $\bar{i}_{k}$.

Remark. Proposed framework takes current limits into account only at the steady-state, so that nothing prevents current $i_{k}$ to exceed $\bar{i}_{k}$ during transient. Indeed, upper bound $\bar{i}_{k}$ translates thermal capacity of electrical components, so that overtaken those limits for a short period of time will not change dramatically temperature of the devices and, hence, is fully acceptable. This suggests that imposing $i_{k}(t) \leq \bar{i}_{k}$ at all time is not only useless but also conservative, in the sense that performances could be lowered by this restriction.

\subsection{Experimental setup description}

The experimental setup, represented on Fig. 15, is composed of two buck converters $(m=2)$, which are heterogeneous in the sense that inductors as well as transistors are different. It happens that electrical components of converter 2 have lower quality but its diode threshold voltage is lower. As a result, inequalities $r_{1,1}=0.1301 \Omega<r_{1,2}=0.3058 \Omega$ and $r_{2,1}=0.3685 \mathrm{~V}>r_{2,2}=0.0361 \mathrm{~V}$ hold and, in turn, induce inversion between converter having priority, depending on the load. This kind of configuration can appear when a fast synchronous buck converter with low power rating is used for converter 2 and a slower classical buck converter with higher power rating is used for converter 1. Characteristics of this setup are listed by Table 2 .

\begin{tabular}{|c|c|}
\hline Parameters & Values \\
\hline \hline Voltage reference $v_{\mathrm{r}}$ & $12 \mathrm{~V}$ \\
\hline Input voltage of the converters $E_{k}$ & $24 \mathrm{~V}$ \\
\hline Switching frequency $f_{s}$ & $20 \mathrm{kHz}$ \\
\hline Sampling frequency & $10 \mathrm{kHz}$ \\
\hline Transistor MOSFET 1 & STP31510F7 \\
\hline Transistor MOSFET 2 & STP30NF10 \\
\hline$L_{1}$ & $1.3 \mathrm{mH}$ \\
\hline$L_{2}$ & $0.6 \mathrm{mH}$ \\
\hline$C$ & $40 \mu F$ \\
\hline
\end{tabular}

Table 2: Experimental Setup Parameters.

The controller hardware is Rapid Control Prototyping (RCP) system dSpace MicroLabBox which is composed of a real-time processor that communicates with an FPGA and several (digital and analog) inputs/outputs. The control law is implemented numerically in the real-time processor (NXP QorIQ P5020, dual-core $2 \mathrm{GHz}$ ). It delivers duty cycles $d_{k}$ to a FPGA (Xilinx® Kintex®-7 XC7K325T FPGA) in charge of the PWM implementation. The binary output signals are conveyed by optical fiber to the driver of transistors (switching cells). Voltage and currents sampling instants are defined according to 
instantaneous value of PWM signals: Measurements are performed at instant times where instantaneous value of the signal equals its average value over one period. This way, average values of voltage and currents are seemingly sampled. Load variations are controlled by the dSpace board through a programmable DC electronic load BK Precision 8600 series with a maximum power of 150W, so that the total current can vary from 0 to $12 \mathrm{~A}$ at the DC voltage $v=12 \mathrm{~V}$.

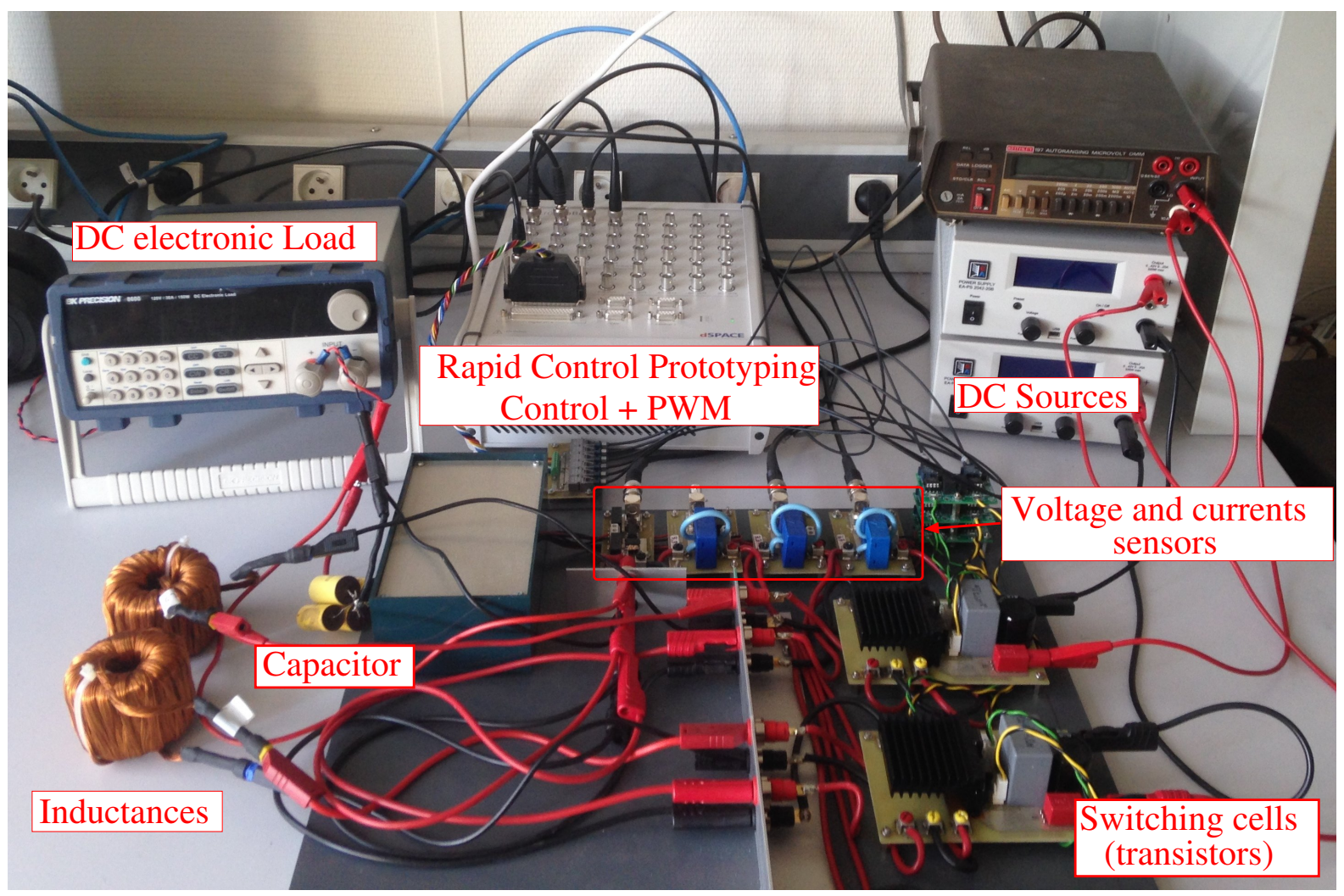

Figure 15: Experimental setup.

\subsection{Solvability assumption}

A single input voltage $E_{1}=E_{2}=24 \mathrm{~V}$ is considered. (i) Regulating voltage stability at the reference $v_{r}=12 \mathrm{~V}$ and (ii) imposing optimal current vector $i_{\text {opt }}$ are control objectives to be achieved for any $R \in \mathbb{L}$. Load set $\mathbb{L}$ reads

$$
\mathbb{L}=[\underline{R}, \bar{R}]=[1.8,12] \Omega
$$

435 and current limits are $\bar{i}_{1}=3.0 \mathrm{~A}$ and $\bar{i}_{2}=4.0 \mathrm{~A}$.

Equality constraints involved in problem (22) impose $v$ equal $v_{r}$ and, from (2b), set $\sigma$ to $\sigma_{r}=v_{r} / R$ (which in turn proves that any feasible point of problem (22) satisfies ASM 1) but let current distribution $\delta_{r}$ free. $^{7}$ In other words, writing problem (22) in the new coordinates leaves us to optimization problem

\footnotetext{
${ }^{7}$ From (2a), also observe that there always exists duty cycles $d_{k}$ equating current derivatives to zero. Steady-state value of $d_{k}$ equals $v_{r} / E_{k}$ and, thus, belongs to [0,1]. This means that, for any physically meaningful voltage reference, i.e. satisfying $v_{r} \leq \min _{k} E_{k}$ (see footnote 1 ), there exists an input vector $d$ satisfying input constraints at the steady-state.
} 
for which decision variables set reduces to $\{\delta\}$, as equality constraints assign values of both $\sigma$ and $v$. 440 See Appendix B for details.

As far as constraint $i \leq \bar{i}$ is concerned, the following chain can be derived:

$$
i \leq \bar{i} \Rightarrow \mathbf{1}_{m}^{\top} i-\mathbf{1}_{m}^{\top} \bar{i} \leq 0 \Leftrightarrow \sigma \leq \mathbf{1}_{m}^{\top} \bar{i}
$$

Last inequality points out that magnitude of total current is limited by $\mathbf{1}_{m} \bar{i}$. As a result, for (22) to admit solution, overall current $\sigma_{r}=v_{r} / R$ to be tracked must but less or equal than $\mathbf{1}_{m}^{\top} \bar{i}$, which, in turn, imposes lower bound on admissible load value.

Assumption 3. The following inequality

$$
v_{r} / \mathbf{1}_{m} \bar{i}=: R_{\mathrm{sat}} \leq \underline{R}
$$

holds.

Note that this assumption is satisfied for considered numerical values.

\subsection{Optimal locus in the $\left(i_{1}, i_{2}\right)$ plan}

Let us now locate reference $x_{r}(R)$ to be reached in the $\left(i_{1}, i_{2}\right)$ plan depicted by Fig. 16 . Note that, when $m=2$, new coordinates $(\delta, \sigma)$ can be easily interpreted by remarking that $(-\delta, \sigma) \in \mathbb{R} \times \mathbb{R}$ simply derives from $\left(i_{1}, i_{2}\right)$ by a rotation of $-\pi / 4$ radian angle follows by an homothetic transformation of ratio $\sqrt{2}$ since

$$
\left[\begin{array}{l}
\Gamma_{2}^{\top} \\
\mathbf{1}_{2}^{\top}
\end{array}\right]=\left[\begin{array}{cc}
1 & -1 \\
1 & 1
\end{array}\right]=\sqrt{2}\left[\begin{array}{cc}
\cos (\pi / 4) & -\sin (\pi / 4) \\
\sin (\pi / 4) & \cos (\pi / 4)
\end{array}\right]
$$

Feasible set. By setting $\sigma$ to $\sigma_{r}=v_{r} / R$, equality constraints of (22) impose that currents has to be stabilized on the dashdot black line ${ }^{8}$ for which $\sigma=i_{1}+i_{2}=v_{r} / R$. Asymptotic value of currents must also belong to the white area, bounded by dashdot red line, where $i \leq \bar{i}$ holds, so that gray area refers to $i>\bar{i}$. The (load dependent) resulting segment, i.e. part of the dashdot black line which belongs to the white area, corresponds to the feasibility set of (22). This is the set where current distribution $\delta$ can be freely adjusted. Note that modifying $R$ results in translating this segment as well as affecting its length. The limit case, for which this length reduces to zero, occurs when the dashdot black line and dot red lines intersect: At this point, each converter provides their maximum current $\bar{i}_{k}$ and $R=R_{\text {sat }}$. Further lowering of the load leads to infeasible problem.

Optimal current distribution. Without loss of generality, assume that converters are numbered in such a way that inequality

$$
\begin{aligned}
& R_{0}:=\frac{v_{r} r_{1,2}}{i_{1}\left(r_{1,1}+r_{1,2}\right)+\left(r_{2,1}-r_{2,2}\right) / 2} \geq \\
& \frac{v_{r} r_{1,1}}{\bar{i}_{2}\left(r_{1,1}+r_{1,2}\right)-\left(r_{2,1}-r_{2,2}\right) / 2}
\end{aligned}
$$

\footnotetext{
${ }^{8}$ This line is nothing but the projection on the $\left(i_{1}, i_{2}\right)$ plan of the subset of $\mathbb{R}^{n}$ satisfying ASM. 1.
} 


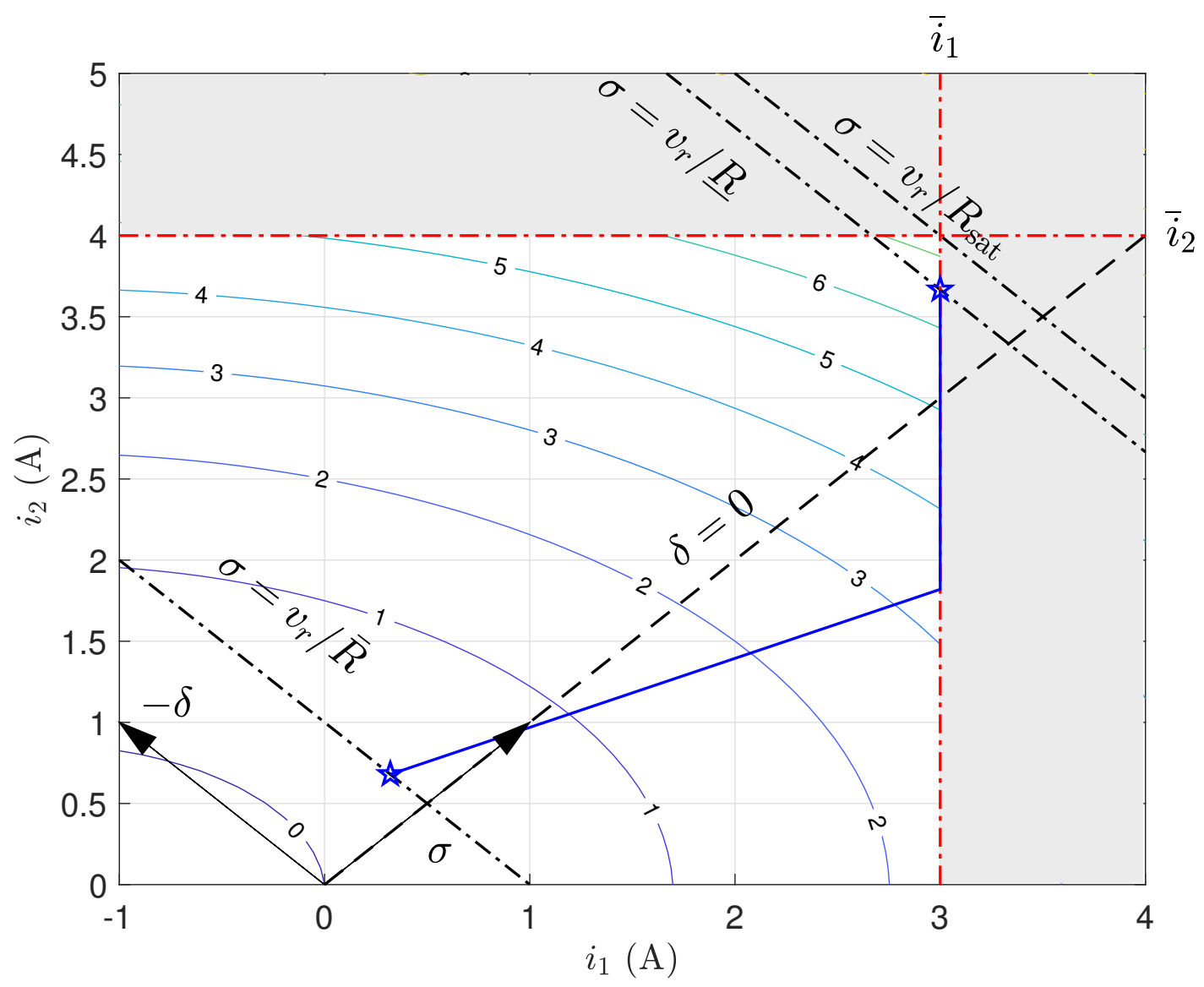

Figure 16: Load profile in the currents plan.

holds. ${ }^{9}$ In such a case, analytical load-dependent solution of (22) can be derived, as shown by Appendix B:

$$
i= \begin{cases}\bar{i}_{1}\left[\begin{array}{c}
1 \\
-1
\end{array}\right]+\frac{v_{r}}{R}\left[\begin{array}{l}
0 \\
1
\end{array}\right], & \left(R \in\left[\underline{R}, R_{0}\right]\right) \\
\frac{1}{r_{1,1}+r_{1,2}}\left(\frac{r_{2,1}-r_{2,2}}{2}\left[\begin{array}{c}
-1 \\
1
\end{array}\right]\right. & \\
\left.+\frac{v_{r}}{R}\left[\begin{array}{l}
r_{1,2} \\
r_{1,1}
\end{array}\right]\right), & \left.\left.(R \in] R_{0}, \bar{R}\right]\right)\end{cases}
$$

Fig. 16 depicts location of this load-dependent optimal steady-state using dashed blue line as well as level lines of $J$ inside the white area. When $R$ belongs to $\left.] R_{0}, \bar{R}\right]$, optimal solution draws a straight line in the interior of the white area where $i \leq \bar{i}$. One recovers the unconstrained expression of optimal current vector provided by [12, Eq. (12)]. When magnitude of $R$ is lower, i.e. $R \in\left[\underline{R}, R_{0}[\right.$, converter 1 reaches its current limit and saturates. In this case, unsaturated converter 2 provides the remaining part of the

\footnotetext{
${ }^{9}$ Indeed, if (25) does not hold, then the resulting inequality is nothing but (25) with $r_{1,1}, r_{1,2}, r_{2,1}, r_{2,2}, \bar{i}_{1}$ and $\bar{i}_{2}$ in place of $r_{1,2}, r_{1,1}, r_{2,2}, r_{2,1}, \bar{i}_{2}$ and $\bar{i}_{1}$ and a strict inequality sign.
} 
total current $v_{r} / R$, required to achieve voltage reference. The fact that converter 1 always hits current boundaries first is due to (25).

Remark (Load-dependent hierarchy). For the considered scenario, it can be seen that $\delta=i_{1}-i_{2}$ changes sign which translates the fact that priority for extraction of electrical power should be given to the first branch $(\delta>0)$ or the second one $(\delta<0)$, depending on load magnitude.

\subsection{Load time evolution}

Load variation with respect to time is defined as the following function:

$$
R(\tau ; t)= \begin{cases}\bar{R}, & \left(0 \leq t \leq t_{1}\right), \\ \bar{R}+(\underline{R}-\bar{R}) \frac{t-t_{1}}{\tau}, & \left(t_{1} \leq t \leq \tau+t_{1}\right), \\ \underline{R}, & \left(\tau+t_{1}<t\right),\end{cases}
$$

which uses parameters $\tau$ as the falling time to reach $\underline{R}$ from $\bar{R}$ and $t_{1}=0.01 \mathrm{~s}$ as starting time for load variation. Hence, transition is increasingly abrupt as $\tau$ decreases. Consequently, lowering parameter $\tau$ tends to increase velocity of the (load-dependent) reference to be tracked by the system, therefore making regulation task more demanding for the control law. If perfect control is expected to drive the system along the broken blue line of Fig. 16, low values of $\tau$ will definitely take system trajectories away from this line.

Three distinct scenarios are considered by affecting values of the following set to $\tau$ :

$$
\tau \in\{0.002,0.005,0.08\}(s),
$$

Upper subplot of Fig. 17 illustrates related chronographs. Resulting time dependent reference $x_{r}(R)$ to be tracked is computed in the new coordinates and $\delta_{r}$ and $\sigma_{r}$ depicted by lower subplots of Fig. 17 (recall that $v_{r}$ does not depend on load and, in turn, has constant magnitude).

\subsection{Experimental results}

Control law is designed by implementing procedure underlined in subsection 5.1 and summarized by Th. 5.3. Resulting controller gains are

$$
\left[\begin{array}{lll}
k_{d} & k_{p} & k_{i}
\end{array}\right]=\left[\begin{array}{lll}
0.237 & -0.174 & -0.061
\end{array}\right]
$$

and $K$ is selected as $-\kappa \mathbf{I}_{2}$ with $\kappa \in \mathbb{R}_{>0}$ in such a way that $\kappa$ governs velocity of current distribution regulation. Here, 5 is affected to $\kappa$. Then, experiments are performed by considering the three load scenarios characterized by $\tau$, successively. Results are represented (i) by Fig. 18 which depicts currents and voltage chronographs and (ii) by Fig. 19 representing both duty cycles and estimated load value. Fig. 20 also allows to visualize those result into the currents plan. From those pictures, the following conclusions can be drawn: (i) For large $\tau$, load estimation as well as voltage and current distribution regulations are sufficiently fast for reference to be accurately tracked, (ii) decreasing $\tau$ pushes closed-loop system to its limit, up to a point where prescribed currents trajectory left out during transient and (iii) 

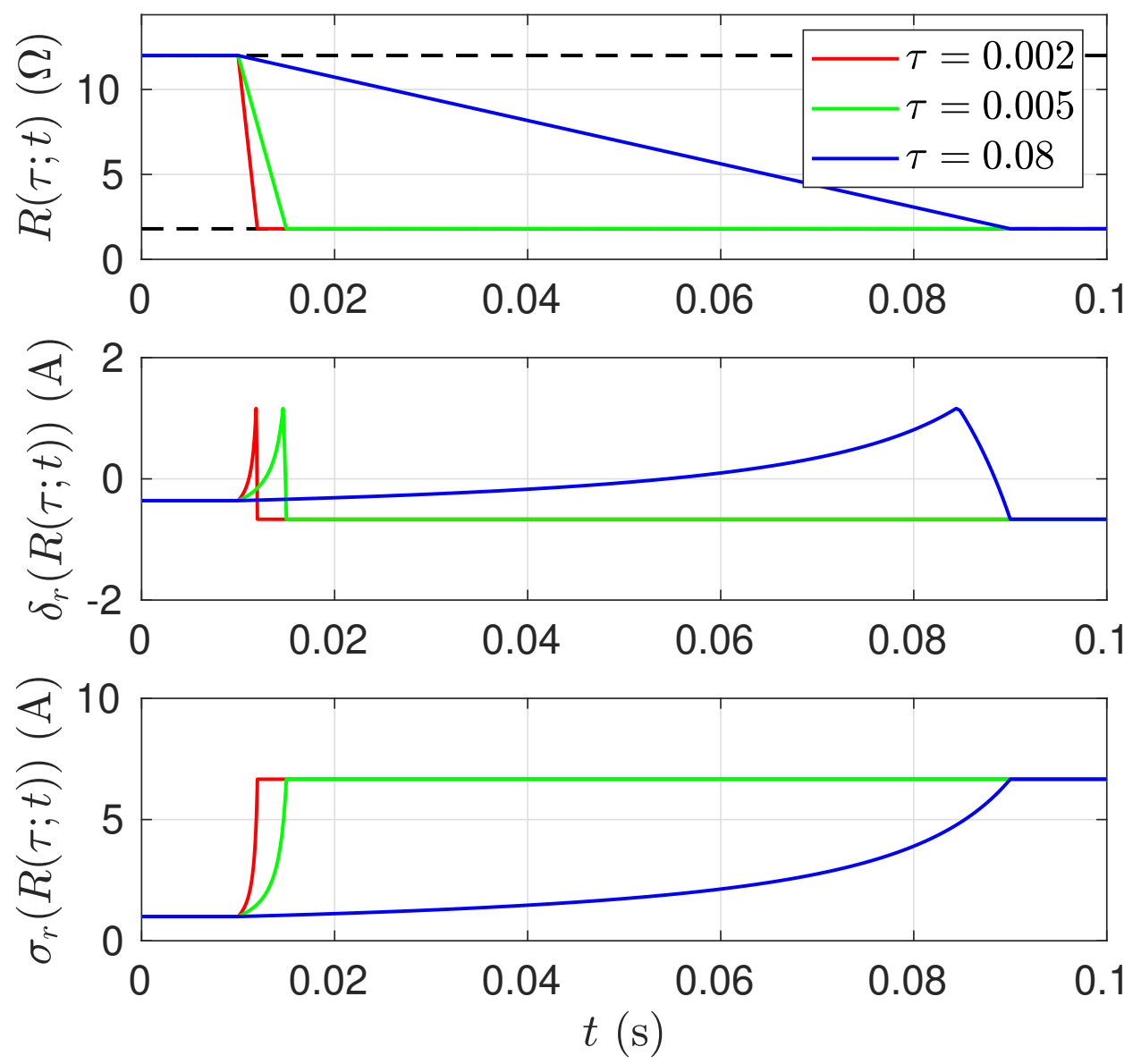

Figure 17: Load and bus voltage chronographs.

stability is preserved for all value of $\tau$. To highlight current ripples, Fig. 21 provides oscilloscope screenshots of the currents and voltage measurements at the steady-state for $R=12 \Omega$ (left) and $R=1.8 \Omega$ (right). It corresponds to zooms on measurements related to Fig. 18, at the beginning and at the end of the timeline.

Remark. Since parasitic elements were not taken into account in design model (2), experimental results suggest that proposed control scheme possesses intrinsic robustness properties to parameter uncertainties resulting from imperfection of electrical components.

\section{Perspectives}

This section discusses possible extensions of this work. Specifically, it aims to evaluate added value of the proposed framework to address questions have been set aside.

\subsection{Constant power load}

In the case where the load cannot be modeled as resistive component but rather as a constant power load (CPL), voltage dynamical relationship (2b) becomes

$$
C \dot{v}=\sigma-p_{0} / v
$$



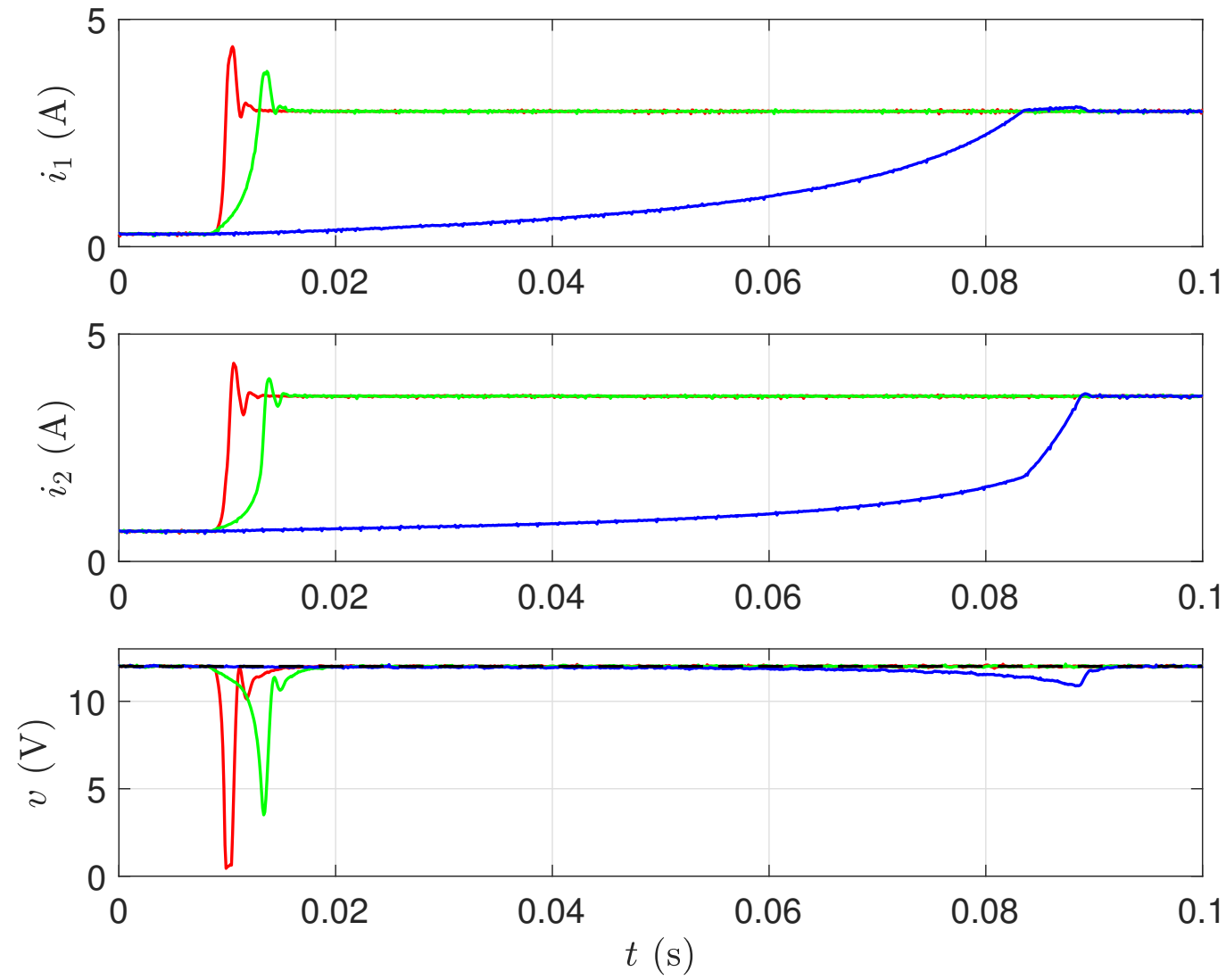

Figure 18: Chronographs of $i(t)$ and $v(t)$. 

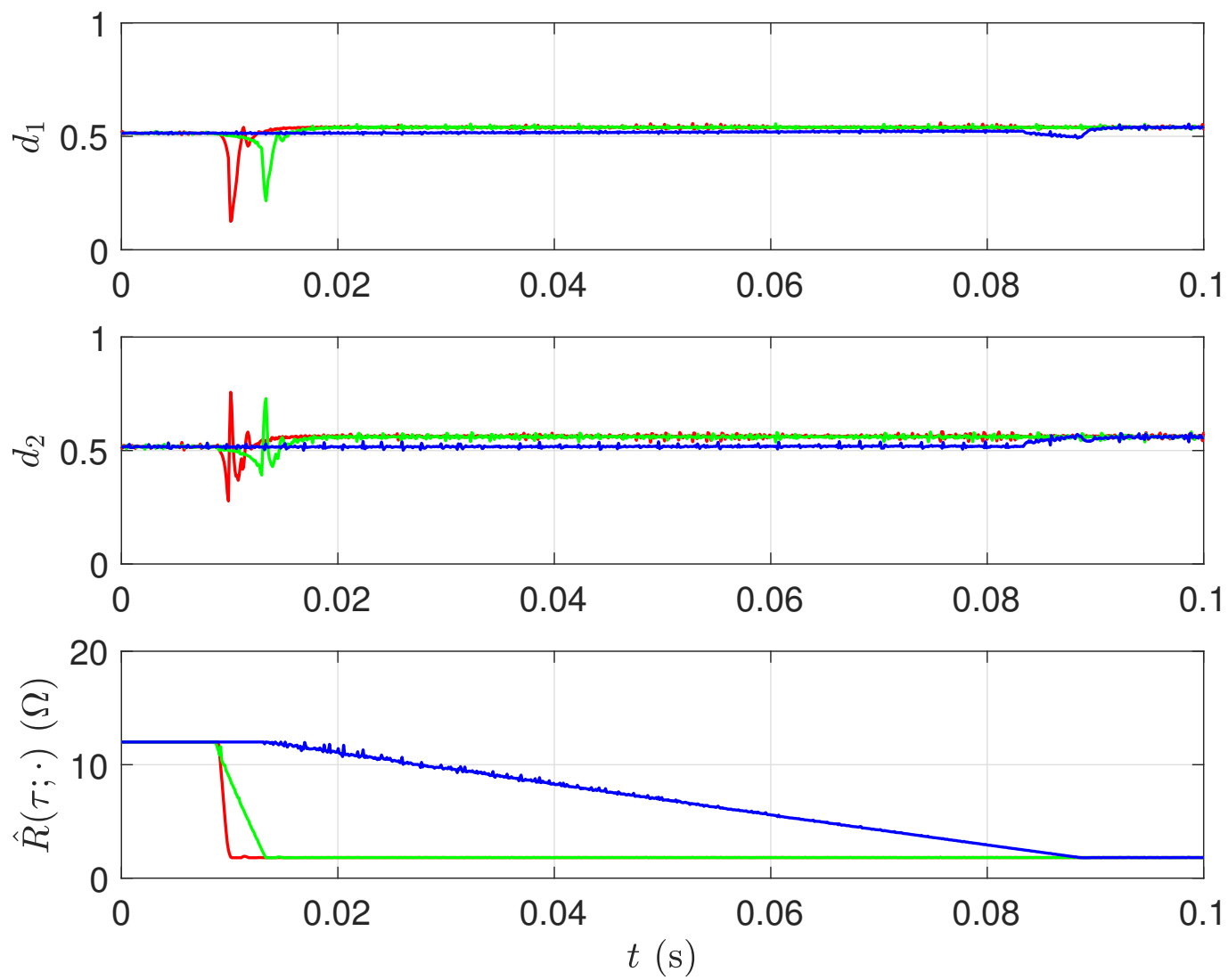

Figure 19: Chronographs of $d(t)$ and load estimation. 


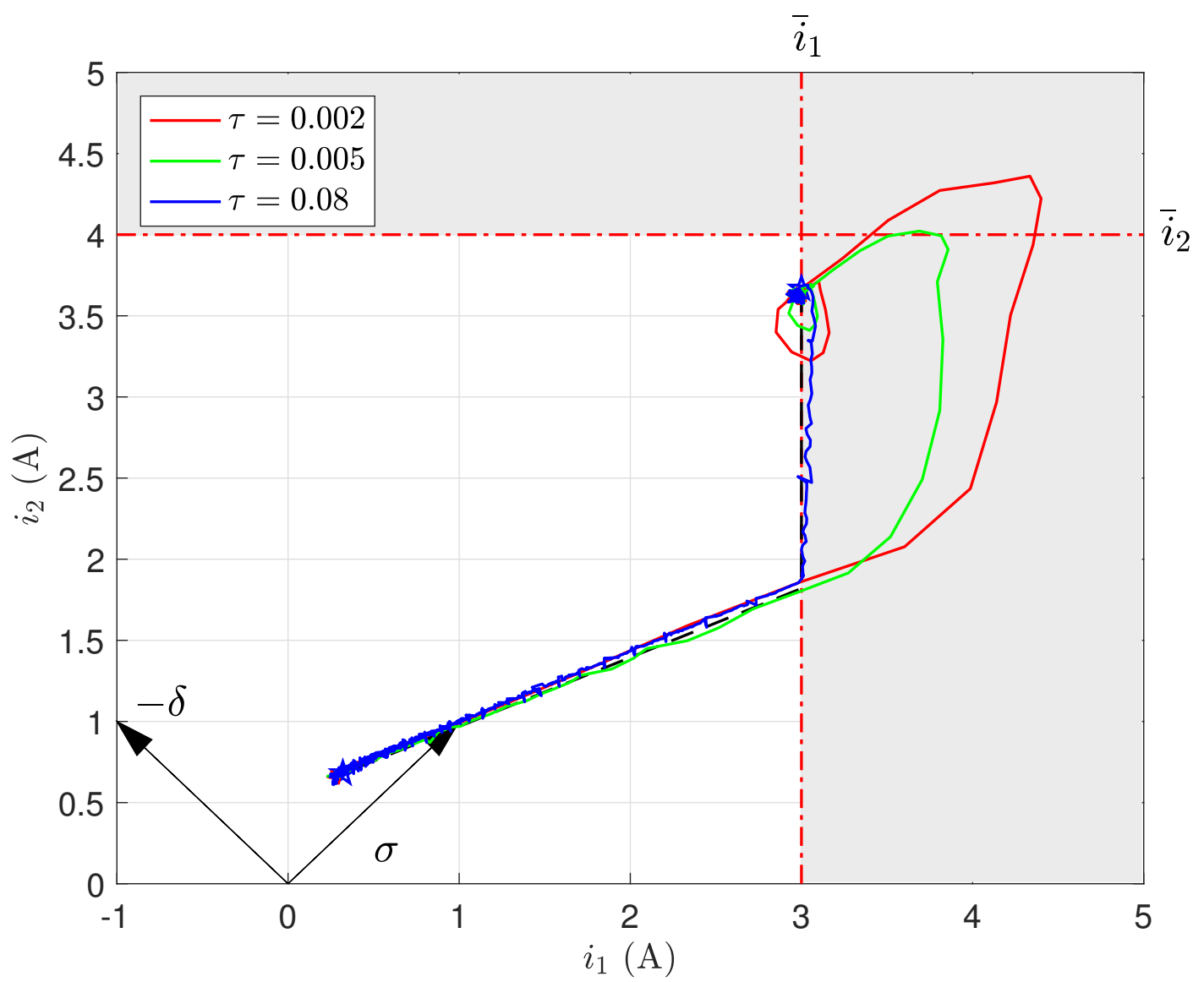

Figure 20: Experimental results in $\left(i_{1}, i_{2}\right)$ plan. 


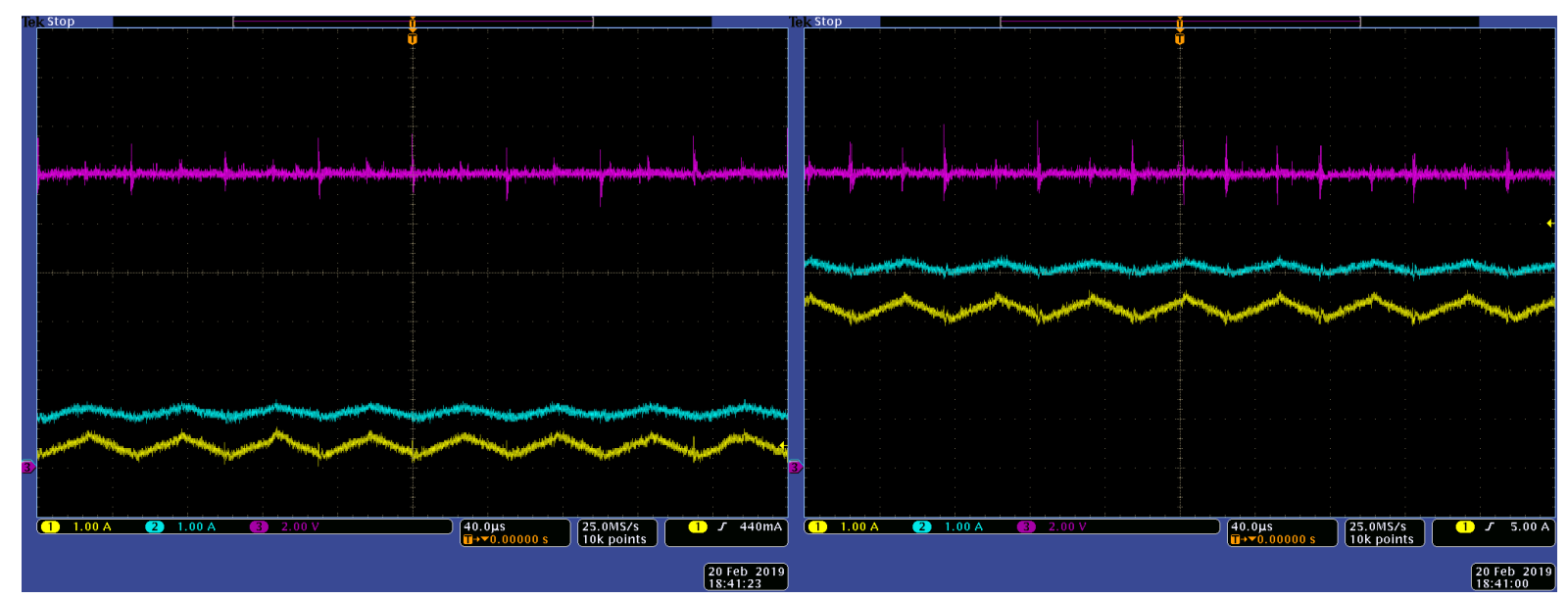

Figure 21: Oscilloscope screen-shots of steady state for $R=12 \Omega$ (left), $R=1.8 \Omega$ (right) ( $i_{1}$ in yellow, $i_{2}$ in cyan, $v$ in magenta).

where term $v / R$ has to be substituted by $p_{0} / v$ with $p_{0} \in \mathbb{R}_{>0}$, see e.g. [23]. This introduces strong nonlinearity that may cause system instability if not properly treated. Nevertheless, the proposed cascaded reformulation allows to isolate this non-linearity to $\Sigma_{v}$ and let $\Sigma_{\delta}$ unchanged. Specifically, lower-right term of state matrix of (14) becomes $-p_{0} /\left(C v^{2}\right)$.

As a result, only $\mathcal{C}_{v}$ delivering $\mu$ has to be modified to cope with this difficulty. Note that the rough load estimator $v_{r} / \sigma$ now reads $v_{r} \sigma$ and asymptotically converges to $p_{0}$, provided that stability of equilibrium satisfying $v_{r}=v$ is ensured by $\mathcal{C}_{v}$.

\subsection{Required measurements}

In this paper current measurements have been assumed to be possible. Indeed, from computation of the rank of the observability matrix, it can be verified that current vector $i$ is not observable from voltage measurement, so that observation techniques cannot be used. ${ }^{10}$

Interestingly, proposed framework can more easily cope with current measurements failure. Indeed, regulation of $\Sigma_{v}$ can also be performed via PI controller using only voltage measurement (see example on Subsection 5.1.1). When implementing such a controller, voltage regulation (the foremost control goal) is ensured to be satisfied if $i$ is not measurable, without any risk of instability. Note however that current distribution is inevitably in open-loop.

\subsection{Saturation of $d$}

With the exception of Section 6 devoted to experimental results, it has been implicitly assumed that $\lambda$ and $\mu$ can be freely assigned. In practice, this is obviously not the case as duty cycle vector $d$ belongs to

\footnotetext{
${ }^{10}$ Note that parasitic elements like internal resistances of inductors induce damping which restore current observability. However, observers relying on this feature are expected to be fragile since magnitude of those parasitic elements are usually badly estimated.
} 
$[0,1]^{m}$. By inverting (11), this constraint can be pre-computed by constructing sets $\Lambda$ and $\Phi$ in which $\lambda$ and $\mu$ has to, respectively, belongs to in order not to saturate $d$. Obviously, $\Lambda$ depends on instantaneous value of $\mu$ and, conversely, $\Phi$ takes $\lambda$ as an input. However, considering that regulation of $v$, associated to $\mu$, is the major control goal, it is reasonable to modify trajectory of $\lambda$ in order to avoid saturation of $\mu$. Saying it differently, by decomposing input vector $d$ into new coordinates $(\lambda, \mu)$, each of them being associated to distinct objectives, we gives a way take hierarchy between control goal into account: Typically would gives priority to $\mu$, governing voltage trajectory, over $\lambda$ regulating current distribution. Giving a detailed answer along those line to input constraints issue is ongoing researches.

\section{Appendix A. Controller expression in the original coordinates}

Using change of coordinates (11) and (5) as well as controller expression (19), one gets

$$
\begin{array}{r}
\operatorname{diag}\{L\}^{-1} \operatorname{diag}\{E\} d=\left[\Gamma_{m}\left(\Gamma_{m}^{\top} \Gamma_{m}\right)^{-1}\right. \\
\times\left[\begin{array}{c}
\kappa\left(\delta_{r}(\hat{R}(\sigma))-\delta\right)+\Delta\left(L^{-1}\right) v_{r} \\
E_{\text {eq }} / L_{\text {eq }} \mu
\end{array}\right] \\
=w(\sigma, \mu)-\kappa \Gamma_{m}\left(\Gamma_{m}^{\top} \Gamma_{m}\right)^{-1} \Gamma_{m}^{\top} i .
\end{array}
$$

It comes out that (20) is nothing but componentwise form of previous relationship. To proves this, observe that

$$
\Gamma_{m}\left(\Gamma_{m}^{\mathbf{\top}} \Gamma_{m}\right)^{-1} \Gamma_{m}^{\boldsymbol{\top}}=\mathbf{I}-\frac{1}{m} \mathbf{1}_{m} \mathbf{1}_{m}^{\top}
$$

can be derived from (8).

\section{Appendix B. Optimal reference expression}

Using (8), problem (22) can be rewritten in new coordinates as follows

$$
\min _{\delta} J\left(\Gamma_{m}\left(\Gamma_{m}^{\mathbf{\top}} \Gamma_{m}\right)^{-1} \delta+\frac{v_{r}}{m R} \mathbf{1}_{m}, v_{r}\right)
$$

$$
\text { s.t. } \quad \Gamma_{m}\left(\Gamma_{m}^{\top} \Gamma_{m}\right)^{-1} \delta+\frac{v_{r}}{m R} \mathbf{1}_{m} \leq \bar{i}
$$

where decision variables set reduces to $\{\delta\}$. Definition of $J$, given by $(23)$, leads to

$$
\min _{\delta} \frac{1}{2} \delta^{\top} Q \delta+q^{\top}(R) \delta+\nu \quad \text { s.t. } \quad A \delta+b(R) \leq \mathbf{0}
$$

for some constant (independent of $\delta$ ) term $\nu$ and with

$$
\begin{aligned}
A:=\Gamma_{m}\left(\Gamma_{m}^{\top} \Gamma_{m}\right)^{-1}, & b(R):=\frac{v_{r}}{m R} \mathbf{1}_{m}-\bar{i} \\
Q:=2 A^{\top} \operatorname{diag}\left\{r_{1}\right\} A, & q(R):=A^{\top}\left(\frac{2 v_{r}}{m R} r_{1}+r_{2}\right)
\end{aligned}
$$

Using KKT conditions and provided that feasilibity set $\mathbb{D}:=\left\{\delta \in \mathbb{R}^{m-1} \mid A \delta+b(R) \leq \mathbf{0}\right\}$ is non empty (which is obviously the case whenever $R \leq R_{\text {sat }}$ holds), this standart problem is known to admit the 
following unique (global) solution (see e.g. [Urruty, p.340])

$$
\delta=-Q^{-1}\left(q(R)+A^{\top} \mu\right)
$$

where Lagrange multiplier $\mu \in \mathbb{R}^{m}$ satisfies

$$
\mu_{k} \geq 0, \quad \mu_{k}(A \delta+b(R))_{k}=0
$$

for all $k \in \mathcal{K}$. In the following, we consider $m=2$.

Suppose that $\left.R \in] R_{0}, \bar{R}\right]$. In this case, let us prove that $A \delta+b<\mathbf{0}$ for $\mu=\mathbf{0}$ and

$$
\delta=-Q^{-1} q=\frac{r_{2,2}-r_{2,1}}{r_{1,1}+r_{1,2}}+\frac{v_{r}}{R}\left(1-\frac{2 r_{1,1}}{r_{1,1}+r_{1,2}}\right),
$$

so that this value of $\delta$ is optimal since (B.2) and (B.3) are trivially satisfied. Define $c(R)$ as follows:

$$
\begin{aligned}
c(R) & :=A\left(-Q^{-1} q(R)\right)+b(R) \\
& =\frac{1}{r_{1,1}+r_{1,2}}\left(\frac{r_{2,1}-r_{2,2}}{2}\left[\begin{array}{c}
-1 \\
1
\end{array}\right]+\frac{v_{r}}{R}\left[\begin{array}{c}
r_{1,2} \\
r_{1,1}
\end{array}\right]\right)-\bar{i} .
\end{aligned}
$$

Then, from the fact that $R \mapsto c_{1}(R)$ is a strictly decreasing function on $\mathbb{R}_{>0}$ (since $r_{1}>\mathbf{0}$ ) and the observation that $c_{1}\left(R_{0}\right)=0$, it follows that $c_{1}(R)<0$ for all $\left.\left.R \in\right] R_{0}, \bar{R}\right]$. In the same way, it can be proved that $c_{2}(R)<0$ on the same interval, since $c_{2}\left(R_{1}\right)=0$ where $R_{1}$ is the right-hand side of (25) so that $R_{0} \geq R_{1}$ holds.

Suppose now that $R \in\left[\underline{R}, R_{0}\right]$. In this case case, let us prove that $A \delta+b \leq \mathbf{0}$ holds and that (B.2) and (B.3) are satisfied for

$$
\mu=\left[r_{2,2}-r_{2,1}+\frac{2 v_{r}}{R} r_{1,2}-2 \bar{i}_{1}\left(r_{1,1}+r_{1,2}\right) \quad 0\right]^{\top}
$$

and

$$
\delta=2 \bar{i}_{1}-\frac{v_{r}}{R}
$$

so that this value of $\delta$ is optimal. First observe that $A \delta+b=\left[0, v_{r} / R-\bar{i}_{1}-\bar{i}_{2}\right]^{\top} \leq \mathbf{0}$ for all $R \geq \underline{R} \geq R_{\text {sat }}$. Then, it remains to show that (B.2) is satisfied and $\mu_{1} \geq 0$ holds. The former can be easily checked, while the latter follows readily from the fact $c_{1}(R) \geq 0$ for all $R \leq R_{0}$ (see discussion above) and by remarking that $\mu_{1}$ equals $2\left(r_{1,1}+r_{1,2}\right) c_{1}(R)$.

Using definition of $T$ provided by (8) and the equality $\sigma=v_{r} / R$ at the steady-state, computation of corresponding expressions of $i$ in both cases leads to (26).

[1] V. J. Thottuvelil, G. C. Verghese, Analysis and control design of paralleled dc/dc converters with current sharing, in: Applied Power Electronics Conference and Exposition, 1997. APEC'97 Conference Proceedings 1997., Twelfth Annual, Vol. 2, IEEE, 1997, pp. 638-646.

${ }_{540}$ [2] Y. Huang, C. K. Tse, Circuit theoretic classification of parallel connected dc-dc converters, IEEE Transactions on Circuits and Systems I: Regular Papers 54 (5) (2007) 1099-1108. 
[3] A. Cid-Pastor, R. Giral, J. Calvente, V. Utkin, L. Martinez-Salamero, Interleaved converters based on sliding-mode control in a ring configuration, IEEE Transactions on Circuits and Systems I: Regular Papers 58 (10) (2011) 2566-2577.

[4] E. Meyer, Z. Zhang, Y.-F. Liu, Controlled auxiliary circuit to improve the unloading transient response of buck converters, IEEE Transactions on Power Electronics 25 (4) (2010) 806-819.

[5] Z. Shan, K. T. Chi, S.-C. Tan, Classification of auxiliary circuit schemes for feeding fast load transients in switching power supplies, IEEE Transactions on Circuits and Systems I: Regular Papers 61 (3) (2014) 930-942.

[6] V. Svikovic, P. A. Cervera, J. A. O. Ramírez, O. G. Suárez, J. A. C. Márquez, Improvement of the dynamic performance of a multiphase current controlled buck converter using an auxiliary synchronous buck converter as an additional energy path, in: XX Seminario Anual de Automática, Electrónica Industrial e Instrumentación SAAEI 2013, Universidad Politécnica de Madrid (UPM) Escuela Técnica Superior de Ingenieros Industriales (ETSII) Servicio de Publicaciones, Madrid, 2013, pp. 0-0.

[7] A. Repton, H. Kobayashi, M. Childs, J. Svorc, Asymmetric inductors in multi-phase dcdc converters, uS Patent App. 14/052,844 (04 2015).

[8] R. Delpoux, J. Trégouët, J. Gauthier, C. Lacombe, New framework for optimal current sharing of nonidentical parallel buck converters, IEEE Transactions on Control Systems Technology (2018) 1-7doi:10.1109/TCST.2017.2787117.

[9] M. Hamzeh, A. Ghazanfari, Y.-R. Mohamed, Y. Karimi, Modeling and design of an oscillatory current-sharing control strategy in dc microgrids, IEEE Transactions on Industrial Electronics 62 (11) (2015) 6647-6657. doi:10.1109/TIE.2015.2435703.

[10] M. Srinivasan, A. Kwasinski, Autonomous hierarchical control of dc microgrids with constant-power loads, in: Applied Power Electronics Conference and Exposition (APEC), 2015 IEEE, 2015, pp. 2808-2815. doi:10.1109/APEC. 2015.7104748.

[11] J. Guerrero, J. Vasquez, J. Matas, L. de Vicuna, M. Castilla, Hierarchical control of droop-controlled ac and dc microgrids ; a general approach toward standardization, IEEE Transactions on Industrial Electronics 58 (1) (2011) 158-172.

[12] J.-F. Trégouët, R. Delpoux, J.-Y. Gaulthier, Optimal secondary control for dc microgrids, in: 2016 IEEE 25th International Symposium on Industrial Electronics (ISIE), Santa Clara, CA, 2016, pp. $510-515$.

[13] S. Luo, Z. Ye, R.-L. Lin, F. C. Lee, A classification and evaluation of paralleling methods for power supply modules, in: Power Electronics Specialists Conference, 1999. PESC 99. 30th Annual IEEE, Vol. 2, IEEE, 1999, pp. 901-908. 
[14] J. Sun, Y. Qiu, B. Lu, M. Xu, F. C. Lee, W. C. Tipton, Dynamic performance analysis of outer-loop current sharing control for paralleled dc-dc converters, in: Twentieth Annual IEEE Applied Power Electronics Conference and Exposition, 2005. APEC 2005., Vol. 2, IEEE, 2005, pp. 1346-1352.

[15] J.-F. Trégouët, R. Delpoux, Parallel interconnection of buck converters revisited, IFACPapersOnLine 50 (1) (2017) 15792 - 15797, 20th IFAC World Congress.

[16] I. Batarseh, K. Siri, H. Lee, Investigation of the output droop characteristics of parallel-connected dc-dc converters, in: Power Electronics Specialists Conference, PESC'94 Record., 25th Annual IEEE, Vol. 2, IEEE, 1994, pp. 1342-1351.

[17] S. K. Mazumder, M. Tahir, K. Acharya, Master-slave current-sharing control of a parallel dc-dc converter system over an rf communication interface, IEEE transactions on industrial electronics (1982) 55 (1) (2008) 59.

[18] P. Seibert, R. Suarez, Global stabilization of nonlinear cascade systems, Systems \& Control Letters 14 (4) (1990) 347-352.

[19] H. Sira-Ramírez, R. Silva-Ortigoza, Control design techniques in power electronics devices, Springer Science \& Business Media, 2006.

[20] C. Olalla, R. Leyva, A. El Aroudi, I. Queinnec, Robust lqr control for pwm converters: an lmi approach, IEEE Transactions on industrial electronics 56 (7) (2009) 2548-2558.

[21] S. Boyd, L. El Ghaoui, E. Feron, V. Balakrishnan, Linear matrix inequalities in system and control theory (1994).

[22] H. K. Khalil, Nonlinear Systems, 2nd Edition, Prentice-Hall, Englewood Cliffs, NJ, 1996.

[23] M. Belkhayat, R. Cooley, A. Witulski, Large signal stability criteria for distributed systems with constant power loads, in: Power Electronics Specialists Conference, 1995. PESC'95 Record., 26th Annual IEEE, Vol. 2, IEEE, 1995, pp. 1333-1338. 